\title{
Reference-free quantification of EEG spectra: Combining current source density (CSD) and frequency principal components analysis (fPCA)
}

\author{
Craig E. Tenke $\mathrm{a}^{\mathrm{a}, \mathrm{b}, *}$, Jürgen Kayser ${ }^{\mathrm{a}, \mathrm{b}}$ \\ ${ }^{a}$ Department of Biopsychology, Unit 50, New York State Psychiatric Institute, 1051 Riverside Drive, New York, NY 10032-2695, USA \\ ${ }^{\mathrm{b}}$ Department of Psychiatry, College of Physicians \& Surgeons of Columbia University, New York, NY, USA
}

Accepted 4 August 2005

\begin{abstract}
Objective: Definition of appropriate frequency bands and choice of recording reference limit the interpretability of quantitative EEG, which may be further compromised by distorted topographies or inverted hemispheric asymmetries when employing conventional (non-linear) power spectra. In contrast, fPCA factors conform to the spectral structure of empirical data, and a surface Laplacian (2-dimensional CSD) simplifies topographies by minimizing volume-conducted activity. Conciseness and interpretability of EEG and CSD fPCA solutions were compared for three common scaling methods.

Methods: Resting EEG and CSD (30 channels, nose reference, eyes open/closed) from 51 healthy and 93 clinically-depressed adults were simplified as power, log power, and amplitude spectra, and summarized using unrestricted, Varimax-rotated, covariance-based fPCA.

Results: Multiple alpha factors were separable from artifact and reproducible across subgroups. Power spectra produced numerous, sharplydefined factors emphasizing low frequencies. Log power spectra produced fewer, broader factors emphasizing high frequencies. Solutions for amplitude spectra showed optimal intermediate tuning, particularly when derived from CSD rather than EEG spectra. These solutions were topographically distinct, detecting multiple posterior alpha generators but excluding the dorsal surface of the frontal lobes. Instead a low alpha/theta factor showed a secondary topography along the frontal midline.

Conclusions: CSD amplitude spectrum fPCA solutions provide simpler, reference-independent measures that more directly reflect neuronal activity.

Significance: A new quantitative EEG approach affording spectral components is developed that closely parallels the concept of an ERP component in the temporal domain.
\end{abstract}

(C) 2005 International Federation of Clinical Neurophysiology. Published by Elsevier Ireland Ltd. All rights reserved.

Keywords: Quantitative EEG (qEEG); Surface Laplacian; Alpha rhythm; Power spectrum; Frequency PCA; Recording reference

\section{Introduction}

\subsection{Quantification of EEG rhythms}

Since Berger's first observations of rhythmicity in the 'resting' EEG, the alpha rhythm has arguably become the best known and most frequently studied EEG pattern (Basar, 1997; Gloor, 1969; Niedermeyer, 1997). For healthy, awake adults, alpha is characterized by a spectral peak at

\footnotetext{
* Corresponding author. Department of Biopsychology, New York State Psychiatric Institute, Unit 50, 1051 Riverside Drive, New York, NY 10032, USA. Tel.: +1212543 5483; fax: +12125436540.

E-mail address: tenkecr@pi.cpmc.columbia.edu (C.E. Tenke).
}

approximately $8-13 \mathrm{~Hz}$ (the classic 'alpha band'), and may reflect neuronal activity related to one or more distinct sources. These sources include the classic posterior 'visual' alpha, a sensorimotor mu rhythm, a temporal 'third rhythm' (Niedermeyer, 1987, 1997), and sleep-related spindle activity (Ishii et al., 2003). Evidence from animal models suggests that alpha rhythmicity is a result of both the tuning of the local cortical network (e.g. Lopes da Silva, 1991; Steriade et al., 1993; Timofeev et al., 2002), as well as the synchronous activation of thalamocortical projections via the thalamic reticular nucleus (Buzsaki, 1991; Steriade, 2000).

Currently, the standard approach to study EEG rhythms uses EEG power spectra as a quantitative measure of 
the variance of the EEG (Bendat and Piersol, 1971; Gasser and Molinari, 1996; Pivik et al., 1993; Pollock et al., 1991). This information is commonly reduced even further by integrating across normative spectral bands (e.g. $8-13 \mathrm{~Hz}$ for alpha). These methodological norms provide operational definitions of band-limited activity that are easily applied to groups of subjects, regardless of the presence or absence of strong rhythmicity in any EEG channel.

Quantitative EEG (qEEG) measures are frequently applied in clinical research to compare spectral topographies recorded from individuals with identifiable pathology with those from a normative database (e.g. Duffy et al., 1981, 1994). Since this approach is largely descriptive, it does not explicitly require a theoretical rationale regarding the nature of the underlying pathology. Instead, statistically reliable differences themselves provide the means for classifying EEG rhythms and topographies. This approach is limited by the characteristics of the normative group, as well as by the quality, stability and sensitivity of the underlying EEG differences. A methodological variation, which relies on selected recording sites to evaluate specific regional hypotheses (e.g. Davidson and Fox, 1989; Henriques and Davidson, 1997), has attracted considerable interest in research on frontal EEG asymmetry and affect in both healthy and psychiatric populations (e.g. Allen and Kline, 2004). The field of Brain Computer Interface (BCI) technology provides convincing evidence for the utility of a targeted, regional approach to qEEG (Babiloni et al., 2001; Cincotti et al., 2003; Pfurtscheller, 2003; Pineda et al., 2003; Wolpaw and McFarland, 1994), since only stable, reliable EEG changes are suitable as a response interface for neurologically impaired patients.

Each of these approaches may be appreciated from a pragmatic, result-driven perspective. However, regional hypotheses may be evaluated more efficiently if the information contained in a complete scalp topography is fully exploited, and even a useful, reproducible BCI measure can generally be improved once its origin is thoroughly understood. Unfortunately, only limited anatomical and physiological inferences can be drawn from EEG spectra. Bridging the gap between the neuronal generators and the observed EEG spectra requires a thorough understanding of the strengths and limitations of spectral methods in the context of a volume-conduction model.

\subsection{Volume-conduction and CSD}

CSD is a reference-independent measure of the strength of extracellular current generators underlying the grossly recorded EEG that is firmly based on a linear volumeconduction model (Nicholson, 1973; Nicholson and Freeman, 1975). This measure can be derived from a vector form of Ohm's law:

$J=\sigma E$ where $\boldsymbol{J}$ is the current flow density, $\boldsymbol{E}$ is the electric field and $\boldsymbol{\sigma}$ is the conductivity tensor of the medium. The application of a divergence operation $(\nabla \cdot)$ allows this formal relationship to be expressed in scalar terms

$I_{\mathrm{m}}=-(\nabla \cdot \boldsymbol{\sigma}(\nabla \Phi))$

where the $\operatorname{CSD}\left(I_{\mathrm{m}}\right)$ is a scalar quantity that is computed from the negative gradient of the measured field potential $(-\nabla \Phi)$. If tissue impedance is spatially invariant, $\boldsymbol{\sigma}$ may be replaced by a scalar constant $\left(\sigma_{\mathrm{s}}\right)$, yielding Poisson's source equation:

$-I_{\mathrm{m}}=\sigma_{\mathrm{s}} \nabla^{2} \Phi$

CSD is thereby proportional to the second spatial derivative (i.e. Laplacian; $\nabla^{2}$ ) of the measured field potential.

Since CSD is always a macroscopic, volume-based measure (Nicholson, 1973), the spatial scale and the physical model in which Eq. (3) is cast will affect the fidelity of the CSD as a measure of the strength of the underlying neuronal generator. At the lowest scale, that is, on the level of scalp-recorded EEG topographies, surface Laplacian CSD estimates are indices of radial current flow into the skull from (normal to) the underlying neural tissue (i.e. radial current flow; Pernier et al., 1988; also see Yao, 2002, and Oostendorp and Oosterom, 1996, for the relationship between the surface Laplacian and the normal derivative of the potential gradient). Even at this macroscopic scale, surface Laplacians allow cautious inferences about neuronal generators. At the next, intermediate scale, the same topographies may be described using inverse models to infer effective intracranial generators (e.g. equivalent current dipoles, Scherg and von Cramen, 1985, 1986; LORETA, Pascual-Marqui et al., 1994). Although these (non-unique) solutions concisely simplify EEG topographies, the plausibility of putative generators must be evaluated in the context of a realistic physiology (e.g. dipolar generators should be oriented normal to the cortical surface). Finally, at a microscopic scale, CSD profiles derived from intracranial EEG recordings have the unique capacity to dissect the 'cortical dipole' (Lorente de No, 1947; Mitzdorf, 1985) into physiologically meaningful patterns of sublaminar sources and sinks (e.g. Buzsaki et al., 1986; Holsheimer, 1987; Kraut et al., 1985; Mitzdorf, 1985; Nicholson and Freeman, 1975; Schroeder et al., 1992). However, intracranial CSD is limited by its invasive nature, being largely restricted to animal models, which may explain why the relevance of intracranial CSD features to the scalp-recorded EEG may not be obvious to basic and clinically-oriented human electrophysiology.

The different CSD measurement scales and models collectively provide a powerful framework for understanding the EEG. Notably, experience obtained from one scale may provide insights for interpreting data at another. For example, evidence from a surface Laplacian CSD topography, or from a representation of it as an equivalent 
dipole, may both support inferences regarding a particular intracranial generator if, and only if, the generator conforms to known neuroanatomy and physiology (e.g. the location, orientation, time course and physiological significance is appropriate for the region in question). Dipole solutions have advantages if a small number of generators are adequate to explain the data, but the physiological plausibility of each identified source must be supported independently. Moreover, if an inverse solution indicates an equivalent dipole with an implausible location or orientation, the most appropriate and concise simplification is a CSD scalp topography. One example of this is when sharply-localized irregularities in the topography arise from partial field closure (i.e. most of the activity is locally cancelled due to the pairing of dipolar activity, with dipole orientations in opposite directions; cf. Fig. 3 of Tenke et al., 1993, for an intracranial CSD analog).

\subsection{EEG power spectra}

\subsubsection{Signal and noise in EEG power spectrum topographies}

Fourier transformation reversibly maps real-valued, time series data into complex-valued, frequency spectra. Even though linear system properties can be preserved in the frequency domain, EEG rhythms have historically been studied using non-linear simplifications of these methods derived from statistical, random noise models (e.g. Bendat and Piersol, 1971; Gasser and Molinari, 1996; Pivik et al., 1993; Srinivasan et al., 1998; Tenke, 1986). These measures emphasize the average variance (mean squared amplitude) of a signal, without regard to spatial or temporal properties. By Parseval's theorem, total power is identical for temporal and spectral functions comprising a Fourier transform pair.

While neuronal contributions to the EEG are subject to linear superposition based on volume conduction, physiological (non-signal) and technical artifacts (noise) also share these properties which can help to disentangle their sources from the EEG of interest. For example, the spatial topography of eye movement or blink artifacts is consistent with volume conduction across the scalp from the eyes, justifying the use of linear regression methods to remove or attenuate them (Gratton et al., 1983; Semlitsch et al., 1986; Woestenburg et al., 1983). Likewise, muscle artifacts may overlap EEG alpha frequencies across frontal sites (e.g. Davidson, 1988; Goncharova et al., 2003; Lee and Buchsbaum, 1987), but their topographic and frequency signatures, being generally localized to the vicinity of specific muscles (e.g. frontalis and temporalis) and predominantly high-frequency in content, will allow their classification as artifacts. However, none of these identifying topographic properties are preserved when using power spectra, which distort the linear relationship between signals by expressing them as mean squares. This problem is further exacerbated by the use of a subsequent logarithmic transformation (Bendat and Piersol, 1971; Pivik et al.,
1993; Tenke, 1986), which can exaggerate extremely small, but topographically reproducible errors in areas with low EEG power. It is therefore not surprising that EEG alpha asymmetries are more stable over posterior regions, where alpha is prominent and well defined (e.g. Allen et al., 2004a, b; Debener et al., 2000a).

\subsubsection{Impact of the recording reference}

EEG scalp topographies are invariably affected by the choice of a recording reference. While the choice of a 'bad' reference may be obvious for a particular topography (e.g. a frontal or central reference to measure an auditory N100 peak (Simson et al., 1976; Näätänen and Picton, 1987), no physically realizable recording reference scheme is immune to the reference problem, including the (montage-dependent) average reference. The reference problem is further exacerbated when the EEG is quantified using power spectra (Pivik et al., 1993), which may suffer topographic distortion or the reversal of hemispheric asymmetries (e.g. Hagemann et al., 2001). Despite the widespread recognition of these concerns, the implications of the choice of a recording reference on EEG power spectrum topographies are often misunderstood.

Fig. 1 shows a heuristic illustration of the recording reference problem for EEG alpha. In this example, a sinusoidal waveform has a posterior (planar) topography that varies linearly in amplitude over space. Peak amplitude increases from 0 to $+3 \mu \mathrm{V}$ (Fpz to Pz; solid lines in Fig. 1A, negativity up), with identical activity at lateral and midline sites (e.g., $\mathrm{P} 3=\mathrm{Pz}=\mathrm{P} 4 ; \mathrm{C} 3=\mathrm{Cz}=\mathrm{C} 4$ ), except for an asymmetry imposed at midfrontal sites (left-smaller-thanright hemisphere; $\mathrm{F} 3=0.5 \mathrm{Fz}<\mathrm{F} 4=1.5 \mathrm{Fz}$ ). After rereferencing all waveforms to $\mathrm{Cz}$ (dashed lines in Fig. 1A), the asymmetry appears to reverse (the $180^{\circ}$ phase-shifted waveforms are greater at F3 than F4), although the difference waveforms are identical at all sites because they are linearly related (Fig. 1B). In contrast, even though the absolute value transformation produces an identical frontal asymmetry (Fig. 1C), the difference waveforms vary across the topography because the linear, spatial dependency of the original waveforms is lost (Fig. 1D). Similar properties may be shown for power (i.e. squared amplitude), and are also preserved in the frequency domain (i.e. power spectra).

Acknowledging these problems, some investigators routinely compare findings using two or more reference schemes (e.g. Bruder et al., 1997; Henriques and Davidson, 1990; Reid et al., 1998; Shankman et al., 2005), based on the implicit rationale that findings are more likely to be valid if results are consistent for various reference schemes. However, there is no a priori assurance that a replication based on any equally arbitrary reference will improve the validity and/or interpretability of the reported findings.

\subsubsection{Spectral analysis of CSD waveforms}

Power spectra computed from CSD waveforms provide a reference-free representation of the current generators underlying the EEG, as well as a concise description of EEG 
A
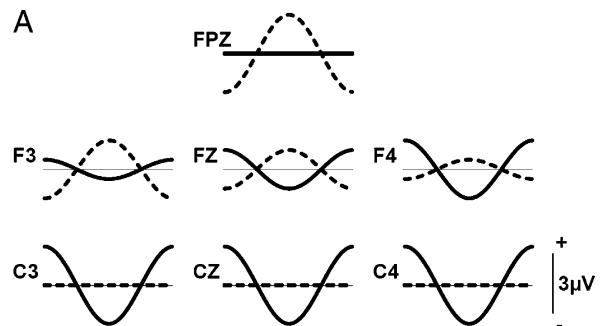

P3-.

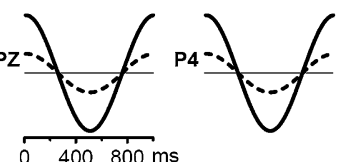

C
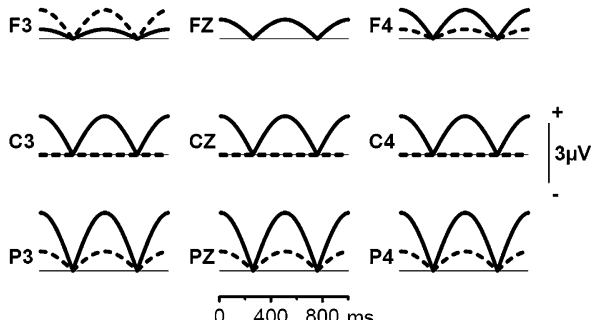

B

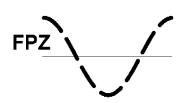
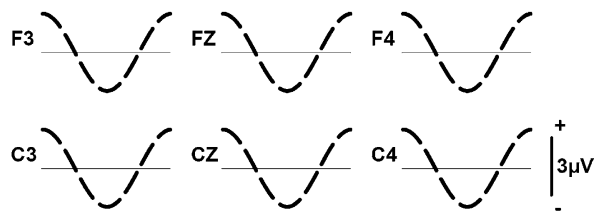

$\frac{1}{1}{ }^{\prime}$
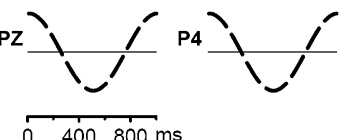

D
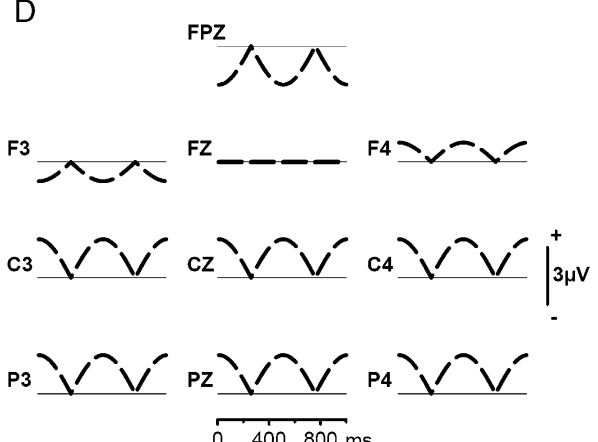

Fig. 1. (A) Arbitrary sinusoidal signal (solid lines) with an amplitude topography varying linearly along the midline from zero at FPz to a maximum peak of $3 \mu \mathrm{V}$ at Pz. Amplitudes of the sinusoid at lateral locations are identical to those at the midline, with the exception of an imposed asymmetry at F3/4 (right greater than left). Rereferencing the sinusoid to $\mathrm{Cz}$ reverses the frontal asymmetry (dashed lines). (B) Differences of waveforms shown in A. Because rereferencing is a linear operation, differences between the original and the rereferenced signals are identical at all sites. (C) When the waveforms shown in $\mathrm{A}$ are rectified, the transformed waveforms maintain properties of the original sinusoidal signal, including the reversed asymmetry at F3/4. (D) The difference between rectified waveforms changes considerably across the montage, because rectification is not a linear transformation.

variance after the removal of volume-conducted activity (Hagemann, 2004; Nunez, 1990; Nunez et al., 1994; Pivik et al., 1993) ${ }^{1}$. CSD alpha spectrum topographies are consistent with those of posterior EEG alpha, including similar hemispheric asymmetries (Hagemann, 2004). CSD power spectra have also been successfully used to study task-related EEG changes (e.g. event-related synchronization and desynchronization; Babiloni et al., 2004). Although CSDs are more sensitive to higher spatial frequencies (e.g. Srinivasan et al., 1998), and may be somewhat less stable than EEG topographies (Burgess and Gruzelier, 1997), even lowresolution CSD estimates may nevertheless be well-suited to represent important properties of generators underlying the surface EEG (e.g. Babiloni et al., 2001, 2002; Kayser and Tenke, in press b; Tenke et al., 1998, 1993).

\footnotetext{
${ }^{1}$ It must be noted that measures based on a Laplacian estimate computed from an EEG power spectrum topography have no formal relationship to Eq. (3), due to the non-linearity of the power spectrum. This criticism also applies to computational simplifications such as the local Hjorth algorithm (Hjorth, 1975), which reduces the Laplacian estimate to a selective, signed sum of local difference potentials (i.e. first derivative estimates). Measures using such approaches (e.g. 'cordance'; Leuchter et al., 1994; Cook et al., 1998) retain the edge-detecting properties of the algorithm, but without regard to the biophysics of volume conduction.
}

\subsection{Frequency principal components analysis ( $P$ PCA)}

A final limitation of standard quantitative EEG methods is the reliance upon a priori frequency bands over which signal estimates are integrated or averaged. Using principal components analysis (PCA) and related methods, pioneering investigations of the spectral structure of EEG variance verified the inadequacy of a single frequency band to characterize alpha activity (e.g. Andresen, 1993; Andresen et al., 1984). Consequently, it is common for investigators to adjust traditional frequency bands or to divide them into subbands so as to assure that spectral peaks are adequately represented, rather than the peaks' rising or falling slopes (Bruder et al., 1997; Davidson and Fox, 1989; Shankman et al., 2005). However, rectangular frequency bands are nevertheless inadequate to accurately quantify overlapping spectral waveforms, a problem precisely comparable to the oversimplification of event-related potential (ERP) components using time windows (e.g. Donchin, 1966; Donchin and Heffley, 1978; Glaser and Ruchkin, 1976; Kayser and Tenke, 2003, 2005).

Although PCA has been used with EEG spectra, this methodology never achieved the degree of acceptance that it had for ERPs, and remained more a descriptive than analytic tool (e.g. Andresen, 1993; Arruda et al., 1996; Duffy et al., 
1994). Moreover, the various methodological debates among ERP researchers about the usefulness of PCA (e.g. Wood and McCarthy, 1984; see Kayser and Tenke, 2005, for a brief historical overview) may have dissuaded many in the EEG community from advocating what was viewed as an unproven and counterintuitive technique. However, the application of PCA to ERPs has been shaped by the construct of an ERP 'component' within the time domain, which requires an identifiable neuroanatomical origin (i.e. characteristic topography) having a distinct time course (i.e. temporal pattern) that can be shown to vary as function of experimental manipulation (e.g. Kayser and Tenke, 2003, 2005; Picton et al., 2000). By analogy, a spectral 'component' would also require a characteristic topography with a distinct spectral shape (i.e. frequency pattern) that can be experimentally manipulated. Under such a definition, the phenomenon of alpha blocking could be considered a rudimentary spectral component (frequency specificity 8$13 \mathrm{~Hz}$, posterior topography, responsive to eyes closed/ open). In contrast, the concept of classical frequency bands explicitly meets only one these requirements (i.e. a frequency range), relieving qEEG researchers from the obligation to characterize the other two (i.e. topographic specificity and condition-dependency). In the absence of an explicit construct to guide the use of spectral methodology, the implications of a spatially-undersampled montage or the lack of an experimental condition verifying the prominence or responsivity of a given spectral measure may not appear to be as relevant.

The recent common availability of more complete recording montages, exceeding the 10-20 standard, and the easy access to considerable computing power has resulted in a renewed interest in PCA for ERPs (Kayser and Tenke, 2005). Analogous to temporal ERP data (Kayser and Tenke, 2003), PCA methodology can overcome the limitations of rectangular spectral windows by defining spectral component waveforms that conform to the underlying data. Whereas treatment-related variance in ERPs may be observed in the amplitude variations of individual components, the simplest analog for resting EEG spectra is the difference in alpha between eyes-open and eyes-closed periods. An additional shift in emphasis from using conventional signal variance (i.e. power spectra) to amplitude spectra and their unique topographies may further facilitate inferences about the neurophysiological substrate of spectral components, if they exist. Preliminary results with this new approach, which has been termed frequency PCA (fPCA; Kayser et al., 2000) in analogy to the terms temporal and spatial PCA used for ERP data analysis, have been promising (Debener et al., 2000b; Kayser et al., 2000).

The argument that solutions based on CSD amplitude spectra are more direct reflections of neural activity than those based on CSD power spectra is not an empirical finding, but rather a deduction from the following facts: (1) the relationship between the grossly recorded EEG and its underlying neuronal current generators may be expressed using a linear, volume-conduction model (Eq. (1)); (2) CSD is a linear, reference-free transformation; (3) Fourier transformation is also a linear transformation; (4) a power transformation is not linearly related to amplitude (i.e. its squared amplitude); (5) PCA preserves the linear properties, since it also represents a linear solution. Given all these facts, amplitude measures are simply more directly interpretable as activity from localizable neuronal generators.

In the present study, we used fPCA to extract concise, data-driven spectral components of relevance to the underlying neuronal generators of the resting EEG in a large sample of human adults. To preserve information about the current generators underlying the EEG, referencefree CSD fPCA solutions were computed to explore their utility for both EEG description and artifact removal, their relationship to standard spectral bands, their stability across samples, and their physiological interpretability. A secondary goal was to directly compare CSD fPCA solutions with nose-referenced EEG fPCA solutions based on common spectral transformations. In accordance with convention, Fourier spectra of EEG and CSD epochs were squared and averaged to form power spectra, which were subsequently simplified as root mean squared (RMS) amplitude spectra, and converted to log power spectra as the de facto standard. Amplitude, power, and log power spectra of CSD and EEG data were submitted to PPCA, and solutions were evaluated using the concept of a spectral component, with a special emphasis on the alpha rhythm.

\section{Methods}

\subsection{Participants}

Resting EEG data collected from 145 adults, pooled across two separate studies (73 male [50.3\%]; age 18-64 years $[M=34.0 ; \mathrm{SD}=9.5]$; ethnicity: 92 Caucasian, 21 African-American, 32 mixed or other ethnic background), were reanalyzed for the current study. Participants consisted of 51 healthy adults and 94 clinically depressed, unmedicated outpatients (for screening details, see Bruder et al., 2002). Individuals were excluded if they had a history of neurological or substance abuse disorder, and were paid US $\$ 30$ for participation. As indicated by the Edinburgh Handedness Inventory laterality quotient (LQ; Oldfield, 1971), participants were mixed in handedness $(M=67.1$, $\mathrm{SD}=49.6 ; n=99$, strongly right-handed, LQ $>70 ; n=13$, left-handed, LQ $<0$ ). The study was approved by the institutional review board, and participation was voluntary.

\subsection{EEG recording and preprocessing methods}

Scalp EEG was recorded from 13 lateral, homologous pairs of electrodes (FP1/2; F3/4; F7/8; FC5/6; FT9/10; C3/4; T7/8; CP5/6; TP9/10; P3/4; P7/8; P9/10; O1/2) and from 
four midline electrodes $(\mathrm{Fz} ; \mathrm{Cz} ; \mathrm{Pz} ; \mathrm{Oz})$ using standard 1020-system placements (Pivik et al., 1993) with an electrode cap (Electro Cap International, Inc.) and a nose reference. Electrodes at supra- and infra-orbital sites surrounding the right eye recorded blinks and vertical eye movements (bipolar), while electrodes at right and left outer canthi recorded horizontal eye movements (bipolar). All electrodes were tin, with impedances below $5 \mathrm{k} \Omega$. EEG was recorded using a Grass Neurodata system at a gain of $10 \mathrm{k}$ (5k and $2.5 \mathrm{k}$ for horizontal and vertical eye channels, respectively), with a bandpass of 0.1-30 Hz. Only recordings which were free of electrolyte bridges between electrodes were included (Tenke and Kayser, 2001).

Continuous EEG were collected with a PC-based EEG acquisition system (Neuroscan) and sampled at $200 \mathrm{~Hz}$ during four 2-min time periods (order of eyes-open [O] and eyes-closed [C] were counterbalanced as OCCO or COOC across subjects). Subjects were instructed to remain relaxed, but awake, and to avoid eye or body movements during the recording periods. During the eyes-open periods, subjects were also instructed to maintain their focus on a pair of crosshairs in the center of a computer screen. The continuous EEG data were segmented into 1.28-s epochs ( $50 \%$ overlap; $0.78 \mathrm{~Hz}$ frequency resolution). Epochs were rejected if amplifiers clipped. Offset-corrected EEG epochs contaminated by amplifier drift, blinks, lateral eye movements, muscle activity or movement-related artifacts were then excluded from analysis using a rejection criterion of + $100 \mu \mathrm{V}$ on any channel, followed by artifacting under visual guidance using a semi-automated procedure. No additional effort was made to reduce or eliminate residual eye and muscle artifact by linear regression approaches.

\subsection{CSD computation}

Epoched CSD waveforms were computed for each accepted epoch using the spherical spline surface Laplacian method (lambda $=10^{-5} ; 50$ iterations; $m=4$ ) of Perrin et al. (1989, 1990). ${ }^{2}$ These parameters have previously been shown to result in CSD waveforms similar to those of the local Hjorth Laplacian for electrodes near the center of this recording montage (Tenke et al., 1998). The reference electrode was included in the montage at a fixed location on the sphere to improve frontal topography, and to provide additional opportunity to detect generators that volumeconduct widely across the recording montage (Junghöfer et al., 1999; Kayser and Tenke, in press a).

\footnotetext{
${ }^{2}$ CSD waveforms were computed using CSD Converter (developed by JK), a program capable of rapidly transforming continuous, epoched, and averaged data for any EEG montage using a spherical model. The computational engine will be provided as generic MatLab source code in a related report (Kayser and Tenke, in press a). All topographies and animations were created using the same spherical spline interpolation suggested by Perrin et al. $(1989,1990)$, but were expressed as $\mu \mathrm{V} / \mathrm{m}^{2}$ to produce values in the same order of magnitude as the EEG.
}

\subsection{Spectral analysis and fPCA methods}

To determine common sources of variance in the spectral data, averaged spectra were submitted to fPCA derived from the covariance matrix, followed by unscaled Varimax rotation (Kayser and Tenke, 2003). For ERPs, this approach produces distinctive, orthogonal factor loadings and weighting coefficients, which efficiently describes the variance contributions of temporally and spatially overlapping ERP components. The number of factors extracted and retained prior to Varimax rotation was not restricted (e.g. using a Scree test or noise variance; cf. Cattell, 1966; Horn, 1965; Kaiser, 1960), which stabilizes meaningful components, while identifying and removing noise-related components (Kayser and Tenke, 2003). Although several limitations of PCA techniques (e.g. misallocation of variance resulting from component jitter or overlap) are well known and require caution, peak or window-based measures are subject to the very same limitations (e.g. Achim and Marcantoni, 1997; Beauducel and Debener, 2003; Chapman and McCrary, 1995; Dien, 1998; Möcks and Verleger, 1986; Wood and McCarthy, 1984). While it is possible that an oblique rotation might provide a simpler solution set, our choice of an orthogonal rotation is nevertheless parsimonious, in that it preserves the overall independence of the factors (Kayser and Tenke, 2005).

By analogy with ERP methods, fPCA factor scores can be interpreted as weighted frequency band amplitudes if the associated factor loadings are clustered in a narrow frequency range and lack significant secondary loadings at different frequencies (Debener et al., 2000b; Kayser et al., 2000). The correspondence between known EEG rhythms (most notably alpha) and fPCA factors, in terms of spectral tuning, topography and treatment dependency (i.e., eyes closed vs. eyes open), allows the identification of physiologically-relevant factors for further analysis. The same properties effectively define the concept of an ERP component for temporal data (Kayser and Tenke, 2005), which may, as some have argued, be inseparably linked to the topography defined by its underlying neuronal generators (Spencer et al., 1999, 2001). CSD fPCA solutions are therefore more appropriate for characterizing spectral components, since CSD provides a more direct measure of the underlying neuronal activity than EEG.

The power spectrum is a methodological standard for describing the spectral variance properties of a time-series (e.g. Bendat and Piersol, 1971). Comparisons between EEG power spectra typically use a subsequent logarithmic transformation as a 'normalizing' procedure (Pivik et al., 1993; Tenke, 1986). However, in two independent preliminary reports, both using nose-referenced EEG data recorded from right-handed adults $(N=29$, 30-electrode montage, Kayser et al., 2000; $N=138$, 29-electrode montage, Debener et al., 2000b), we reasoned that amplitude scaling would restore proportionality to the amplitude of an underlying sinusoid (i.e. RMS amplitude), 
while preserving standard EEG methods that rely on power spectra (Pivik et al., 1993). For both of these two data sets, fPCA produced interpretable solutions that separated identifiable sources of artifact from the EEG, and included multiple treatment-dependent alpha factors. Additional support for focusing on CSD amplitude as a measure of neuronal activity is provided by Logothetis et al. (2001), who reported that the BOLD fMRI response of visual cortex in monkeys to checkerboard stimuli is correlated with the local field potential, which is, in turn, reflected by the intracranial CSD (Logothetis, 2003). In the present study, we replicated and extended these preliminary EEG findings by comparing EEG and CSD spectra for three different quantification procedures. Mean power spectra were computed separately for epoched EEG and CSD data (1.28-s epochs; $0.78 \mathrm{~Hz}$ resolution; $50 \%$ epoch overlap; $50 \%$ Hanning window) for each participant (145), electrode (31), and condition (eyes open vs. eyes closed). The impact of scaling method was then examined by applying a logarithmic ${ }^{3}$ or amplitude (square root of power) transformation to the power spectrum averages, and submitting each to fPCA using a MatLab function (appendix of Kayser and Tenke, 2003) that emulates the PCA algorithms used by BMDP statistical software (program 4M; Dixon, 1992). Mean EEG spectra $(0-77.2 \mathrm{~Hz} ; 100$ frequency points $=100$ variables) were submitted to unrestricted covariance-based PCA, using electrodes (30) $\times$ conditions $(2) \times$ participants (145) as 8700 cases, followed by unscaled Varimax rotation (Kayser and Tenke, 2003; also see Donchin and Heffley, 1978; Glaser and Ruchkin, 1976). For CSD data, the nose reference electrode was also included (31 electrodes $\times 2$ conditions $\times 145$ participants $=8990$ cases $){ }^{4}$

The distinctiveness and interpretability of factor loadings and averaged factor score topographies produced by each scaling method were compared and contrasted (see also footnote ${ }^{1}$ ). By analogy to a temporal PCA, only physiologically meaningful fPCA components were considered (Kayser and Tenke, 2003). Since alpha activity is the most robust and stable physiological pattern in the resting EEG, only the most distinctive alpha factors were further explored in this study.

The existence of a secondary topography on the frontal midline for one CSD alpha factor made it impossible to

\footnotetext{
${ }^{3}$ Since a covariance-based PCA of $\log$ power spectra eliminates the proportionality of the measure (i.e. the factor scores) at different recording sites by removing the grand mean, differences no longer represent logarithms of ratios. Although this consideration would be of relevance for statistical analyses of the factor scores, it is irrelevant for the present purpose of identifying unique variance contributions in the log-transformed data.

${ }^{4}$ Since the logarithm of zero is undefined, the reference electrode was excluded for all EEG fPCA solutions to allow a direct comparison of the impact of scaling method (i.e. power, log power, and amplitude spectra). However, a comparison of the first eight rotated factor loadings for EEG power spectrum solutions with (31 electrodes) or without (30 electrodes) the (zero-valued) reference were virtually identical.
}

confidently infer an underlying neuronal generator configuration without additional information. For this reason, the possibility of concurrent activity in multiple regions was evaluated by comparing factor score topographies for subgroups based on the prominence of the secondary focus. Because it is not possible to distinguish between statistically independent and phaselocked activity in any of the spectral averages, the association between alpha waveforms in primary and secondary regions was directly evaluated in the time domain. For the purposes of this preliminary report, evidence for phase-locking was derived from individual time epochs showing strong rhythmicity, and from the corresponding coherence spectra.

\section{Results}

\subsection{Comparison of averaged EEG and CSD power spectra}

Nose-referenced EEG power spectra (Fig. 2A) were characterized by a prominent, condition-dependent alpha peak at posterior sites that was superimposed on low frequency activity at all electrodes (the 'peak' at $0.8 \mathrm{~Hz}$ is a result of subtracting the epoch mean). Alpha was broadly distributed, with regional variations in peak frequency and maximal amplitude at the parietal midline (Fig. 2C). Condition-dependent differences paralleled the topography of alpha (Fig. 2A), but were measurable even at the frontal midline (Fig. 2E).

Reference-independent CSD power spectra were also characterized by prominent, condition-dependent alpha peaks with a posterior topography, and the shape of the alpha peak varied considerably across the topography (Fig. 2B and D). However, in contrast to EEG power spectra, alpha activity identified in CSD power spectra had a more restricted topography that was more easily distinguishable from the superimposed low frequency activity.

\subsection{Comparison of EEG and CSD fPCA solutions}

\subsection{1. fPCA solution for EEG power spectra}

Fig. 3A summarizes the PPCA solution derived from EEG power spectra. A unique color is used for each of the first eight factor loading waveforms. For example, the factor with the highest loading peak is plotted as a black waveform, and its prominent peak at approximately $10 \mathrm{~Hz}$ is indicated by a black line connecting the corresponding pair of factor score topographies to the $10-\mathrm{Hz}$ value on the frequency axis (i.e. the third pair from the right; $22.1 \%$ variance; $10.2 \mathrm{~Hz}$ ). Factor score topographies are arranged according to peak frequency in order to facilitate their correspondence to loading peaks (i.e., the sequence of maps is identical to the sequence of waveform peaks). The inset shows the same waveforms, but using an enhanced frequency resolution to accentuate alpha activity. Table 1 
A

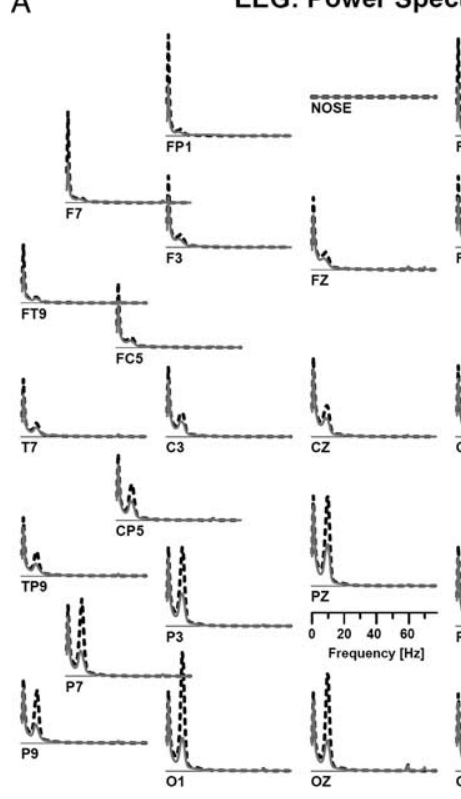

C
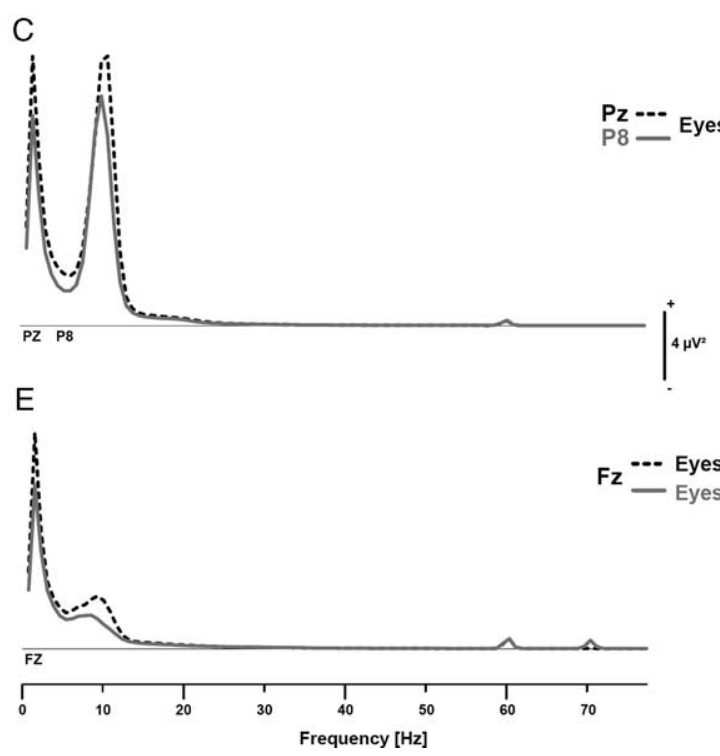

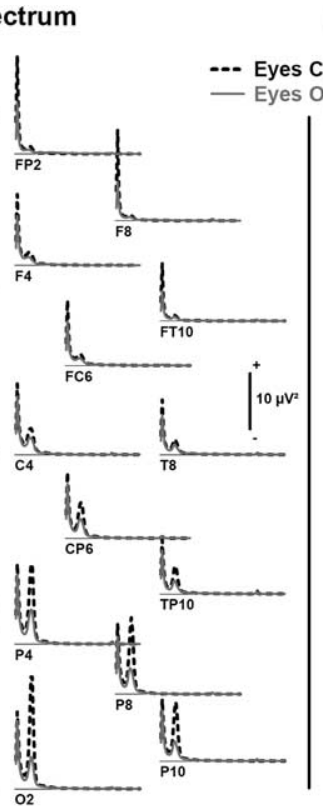

B

CSD: Power Spectrum

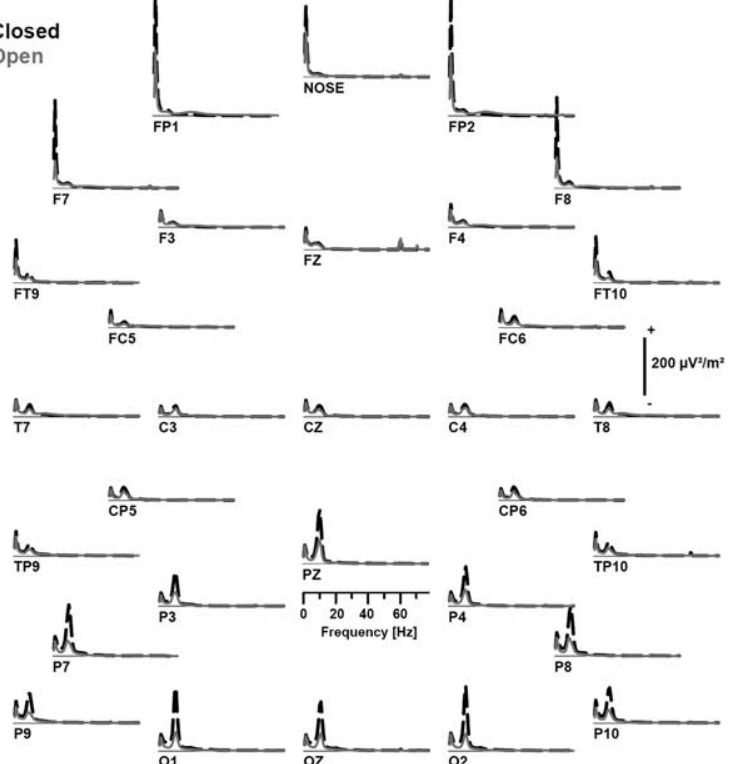

D

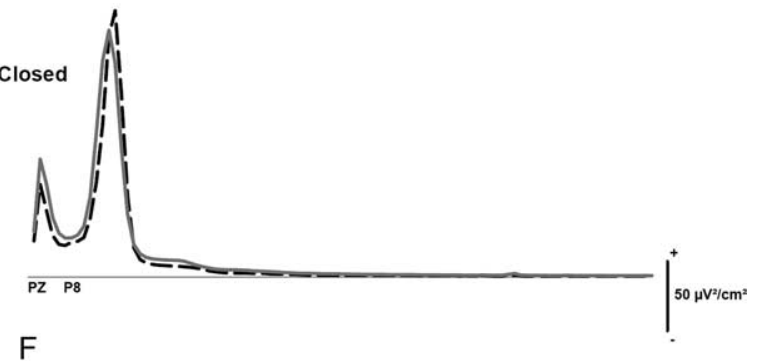

$\mathrm{F}$

--- Eyes Closed
- Eyes Open

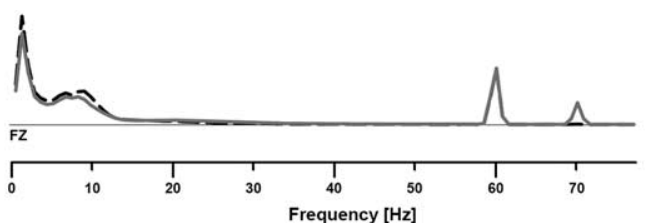

Fig. 2. Grand average power spectra of nose-referenced EEG (A) and reference-free CSD (B) in 145 adults at all 31 recording sites (including the nose tip recording reference) for eyes closed (black dashed lines) and eyes open (solid gray lines) at rest. Enlarged spectra directly compare EEG (C and E) and CSD (D and F) at specific sites: Pz (dashed) and P8 (solid) for eyes closed only (C and D); site Fz for eyes closed (dashed) and eyes open (solid) conditions (E and F).

lists and identifies all eight factors. For example, the first factor is identified as 'alpha', with a medial/posterior topography that is most prominent for the eyes-closed condition.

As summarized in Table 1, six factors accounted for over $90 \%$ of the variance of the EEG power spectra. All six were largest (greater factor scores) for eyes-closed vs. eyes-open recording periods, and five contributed directly to alpha activity (Fig. 3A). Although four of the five alpha factors showed a posterior topography, the topography of the lowest frequency factor $(7.8 \mathrm{~Hz}$ peak) was more anterior and largest on the midline (i.e. from Fz-to-Pz). The remaining factor was consistent with eye artifact $(0.8 \mathrm{~Hz}$ peak, frontopolar topography), despite a secondary topography along the midline and a secondary peak in alpha (Fig. 3A, inset of loadings showing alpha). Overall, the EEG power spectrum fPCA yielded multiple alpha factors with similar or identical peak frequencies, and no evidence of high frequency activity (Fig. 3A).

\subsection{2. fPCA solution for CSD power spectra}

As shown in Table 1, fPCA solutions for CSD power spectra were similar to those for EEG power spectra, producing six factors that accounted for over $90 \%$ of the variance of the spectra, five of which were most prominent for eyes-closed recording periods. However, in contrast to 

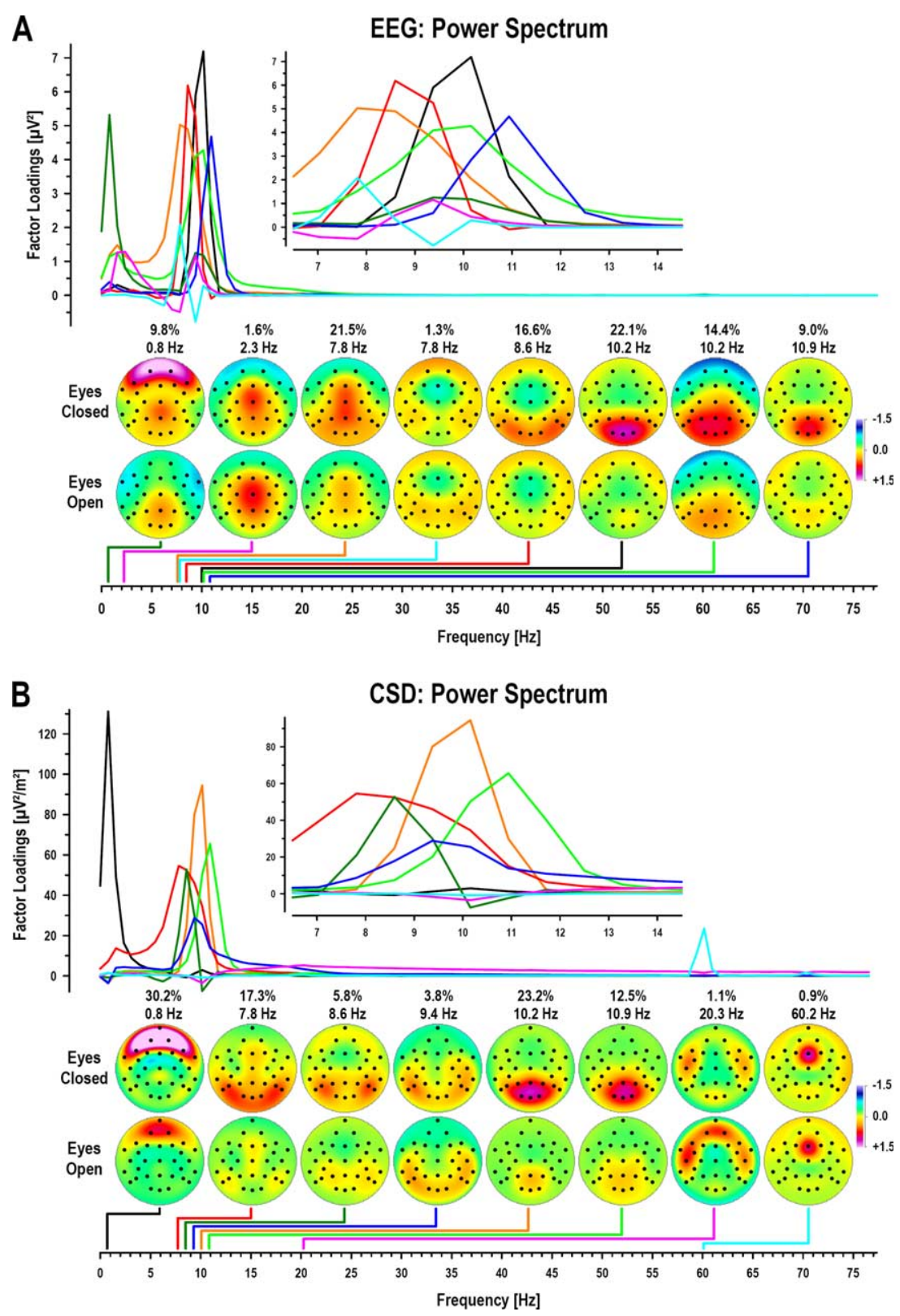

Fig. 3. Comparison of frequency Principal Components Analyses (fPCA) derived from nose-referenced (EEG) and reference-free (CSD) power (A and B), log power (C and D), or amplitude (E and F) spectra, using unrestricted, covariance-based, and Varimax-rotated factor solutions (Kayser and Tenke, 2003). The factor loadings for the first eight components extracted are plotted as overlaid frequency spectra (frequency range $0-77.3 \mathrm{~Hz}$ ) above their associated mean factor score topographies. Insets in A, B, E, and F show enlarged representations of factor loadings for frequencies encompassing the alpha range (8-13 Hz). Mean factor score topographies $(N=145)$ are plotted separately for eyes closed (top rows) and eyes open (bottom rows) conditions. Topographies were sorted according to the factor loadings' peak frequencies (left to right), which are indicated along with the accounted variance above the topographic maps. Black dots indicate the spherical positions of the recording sites (nose at top). All topographic maps are 2D-representations of spherical spline surface interpolations (Perrin et al., 1989, 1990) derived from the mean factors scores available for each recording site. Note the larger scale in C for factor score topographies of EEG log power spectra $( \pm 1.75)$ as compared to the other five solutions $( \pm 1.5)$. Colored lines below maps pointing to the factor loadings' peak frequencies on the abscissae have the same color as the corresponding factors in the factor loadings plot.

EEG power spectra, the highest-variance CSD component consisted of a simpler eye artifact factor $(0.8 \mathrm{~Hz}, 30.1 \%)$, with neither a secondary loading nor a secondary midline topography (see Fig. 3B). All five alpha factors had a posterior topography, but only factors with the lowest peak frequencies $(7.8,8.6$, and $9.4 \mathrm{~Hz})$ included lateral (P7/P8) and inferior (P9/P10) parietal sites. Only one alpha factor
$(7.8 \mathrm{~Hz})$ included a secondary midline topography. Additionally, low variance factors $(<2 \%)$ reflected muscle $(20.3 \mathrm{~Hz}$; broad, low amplitude component) and electrical line $(60.2 \mathrm{~Hz})$ artifacts. Overall, the fPCA solution for CSD power spectra produced factors with greater topographic and spectral specificity than the solution for EEG power spectra. 

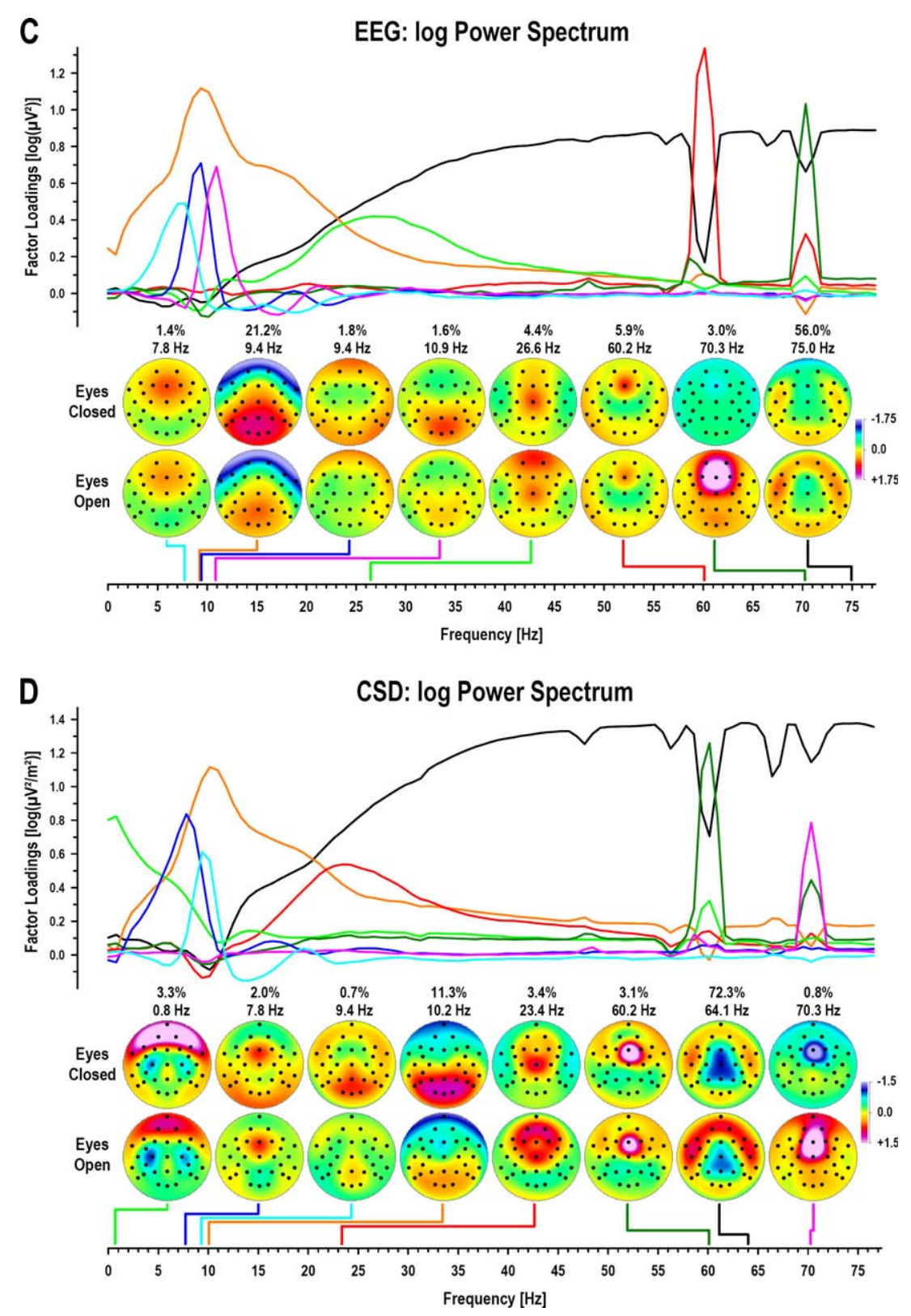

Fig. 3 (continued)

\subsection{3. fPCA solution for EEG log power spectra}

The EEG log power spectrum fPCA solution differed substantially from both power spectrum solutions (Table 1; Fig. 3C). Although five factors accounted for $90 \%$ of the variance of these waveforms, only one represented EEG alpha $(9.4 \mathrm{~Hz} ; 21.2 \%$ variance). This factor was greatest for eyes-closed periods, and had a broad, posterior topography. Although eye artifact was not represented by any of these factors, the highest variance component represented muscle artifact $(75.0 \mathrm{~Hz}, 56.0 \%$, greatest for eyes open). A beta factor $(26.6 \mathrm{~Hz})$ overlapped the time course of the muscle artifact factor and extended its topography medially (central and frontopolar, greatest for eyes open), suggesting that it, also, may represent muscle artifact. The remaining two factors that accounted for more than $2.5 \%$ of the variance represented line $(60.2 \mathrm{~Hz})$ and CRT $(70.3 \mathrm{~Hz})$ artifacts. Thus, the EEG log power solution produced the undesirable outcome of fewer high-variance factors, broader spectral peaks, and greater loadings at high frequencies compared with the EEG power spectra solution.

\subsection{4. fPCA solution for CSD log power spectra}

The differences between the CSD fPCA solution for log power spectra and solutions for the other two spectra were similar to those described for EEG fPCA. Only one of the factors represented alpha activity $(10.2 \mathrm{~Hz}$; Table 1 and Fig. 3D), and muscle artifact was reflected by a high frequency factor $(64.1 \mathrm{~Hz}$, greatest for eyes-open, maximal 

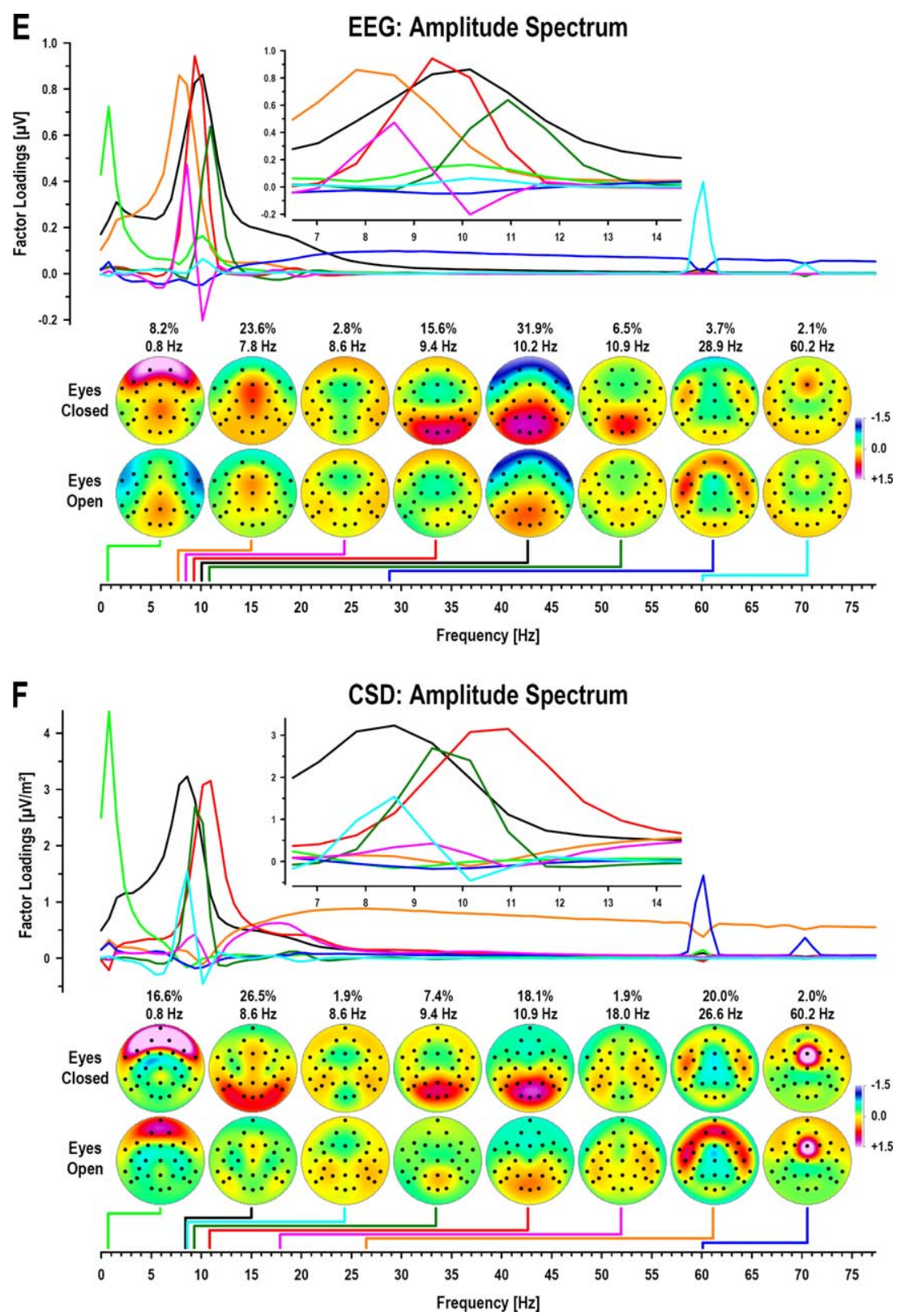

Fig. 3 (continued)

near facial musculature). A second beta factor $(23.4 \mathrm{~Hz})$ was also extracted with an eyes-closed maximum that effectively extended the peripheral topography of the muscle artifact factor into frontal regions. Only four factors were required to account for $90 \%$ of the variance, the last of which was an eye artifact factor $(0.8 \mathrm{~Hz}$; maximum at frontopolar and nose electrodes).

\subsection{5. fPCA solution for EEG amplitude spectra}

Seven factors accounted for $90 \%$ of the variance of the EEG amplitude spectrum (Table 1, Fig. 3E). Five of these factors contributed to alpha (i.e. see loading inset in Fig. 3E). The three high-frequency alpha factors (9.4, 10.2 , and $10.9 \mathrm{~Hz}$ ) had posterior topographies and were greatest for eyes-closed periods, while the lowest frequency alpha factor $(7.8 \mathrm{~Hz})$ had a midline frontocentral topography. The last of these factors also had low variance $(8.6 \mathrm{~Hz}$, $2.8 \%$ ), a loading that included negative values within the alpha band, no condition dependency, and a lateral topography (Fig. 3E), suggesting that it may be a frequency-shifting 'correction' factor, rather than an independent alpha factor. The remaining two factors represented eye $(0.8 \mathrm{~Hz}, 8.2 \%$; included a secondary alpha loading and midline topography) and muscle artifact (28.9 Hz, 3.7\%). When compared to EEG power and EEG $\log$ power solutions (Fig. 3A and C), the fPCA solution for EEG amplitude spectra (Fig. 3E) was intermediate in the density of spectral peaks (spacing of colored lines along 


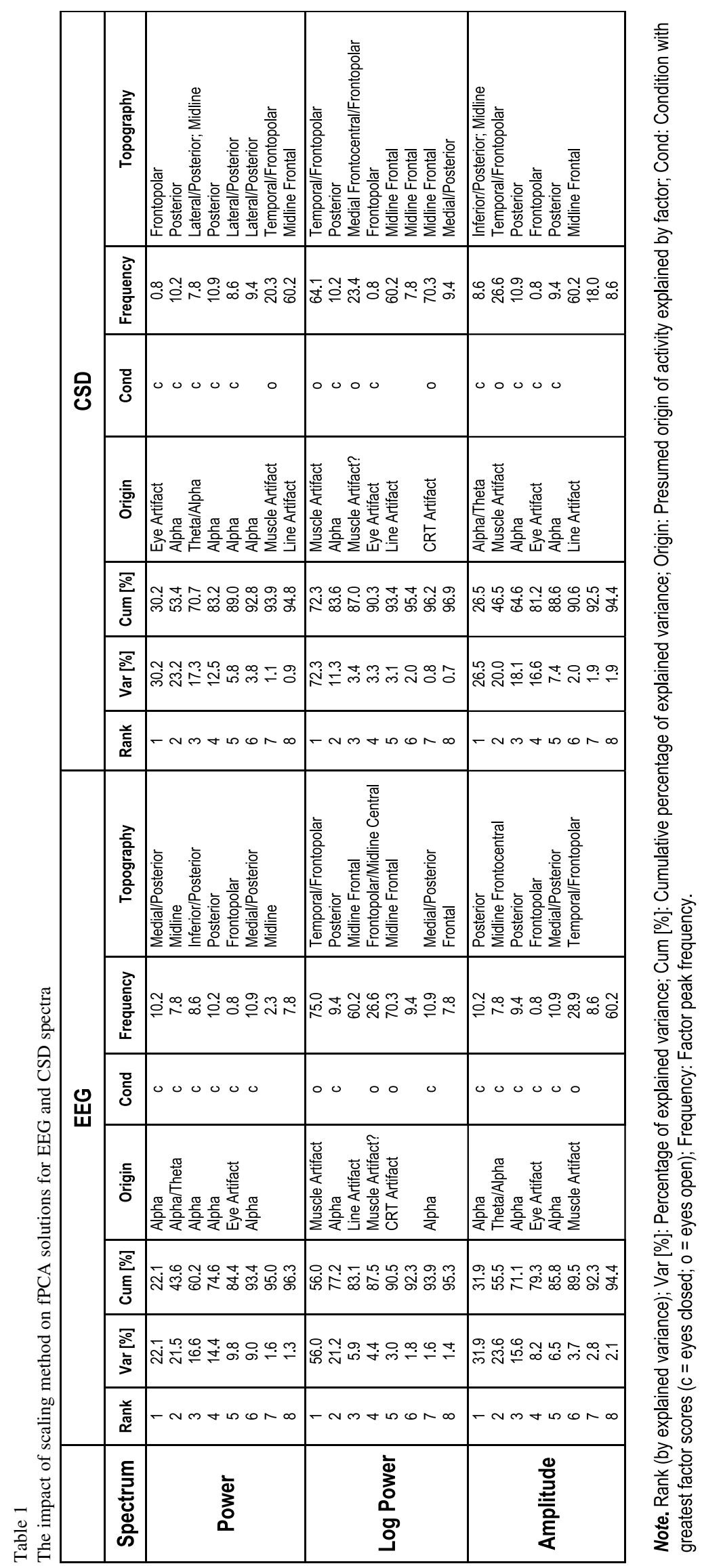


frequency axis) and in the number of alpha peaks ( 5 vs. 6 or 4$)$.

\subsection{6.. fPCA solutions for CSD amplitude spectra}

The CSD amplitude spectrum fPCA solution produced six factors accounting for $90 \%$ of the variance (Table 1 , Fig. 3F). Three of these factors $(8.6,9.4$, and $10.9 \mathrm{~Hz})$ were alpha factors, including the highest variance factor. All three alpha factors had posterior topographies, and were greatest for eyes-closed periods. Eye artifact $(0.8 \mathrm{~Hz}$, $16.6 \%)$ and muscle artifact factors $(26.6 \mathrm{~Hz}, 20.0 \%)$ were both more prominent for this PPCA solution than for either the CSD power spectrum or the EEG amplitude spectrum. The sixth factor was a low variance factor reflecting line artifact $(60.2 \mathrm{~Hz}, 2.0 \%)$.

Although the loading peak for the most prominent factor was within the traditional alpha band $(8.6 \mathrm{~Hz}, 26.5 \%)$, the rising phase of the waveform included a substantial contribution to the classical theta band $(4-8 \mathrm{~Hz}$, Fig. 3F). This factor had a bilateral posterior/inferior topography that was most prominent during eyes-closed periods, as well as a secondary topography extending to the frontal midline, but falling off sharply at frontocentral locations displaced from the midline. In contrast, the high-frequency alpha factor $(10.9 \mathrm{~Hz}, 18.1 \%)$ was distinguishable by its medial parietal topography, its steep topographic fall-off at anterior sites, and its incomplete blockade for eyes-open periods. Compared to the high- and low-frequency alpha factors, the remaining alpha factor had a narrower bandwidth at an intermediate frequency $(9.4 \mathrm{~Hz})$ than the other two alpha factors (inset of Fig. 3F), with a parietal topography that was similar to that of the high alpha factor, but less sharply localized to sites near the midline.

\section{3. fPCA alpha factors from CSD amplitude spectra: reproducibility across groups}

All three of the alpha factors from the CSD amplitude fPCA solution showed reproducible, condition-dependent, posterior topographies across independent samples (Fig. 4A) and groups (healthy controls and depressed patients, Fig. 4B). However, only the low alpha/theta factor $(8.6 \mathrm{~Hz})$ showed a secondary, anterior topography, notably including the frontal midline (electrode Fz). The consistency of these topographies suggests that they represent a stable, physiological process, rather than merely fortuitous variance allocation patterns.

Although the posterior alpha factor topographies are consistent with classical descriptions of the resting alpha rhythm, the existence of a factor straddling the alpha and theta bands poses empirical and theoretical problems. Specifically, it may be questioned whether the identification of the factor as alpha (or theta) is appropriate. Instead, it may be argued that the factor represents at least two distinctive, but unrelated, patterns of activity that vary across subjects, yet are sufficiently similar to be extracted as a single (erroneous) component. In fact, task-specific theta
A Consistency of Alpha Topographies Across Studies

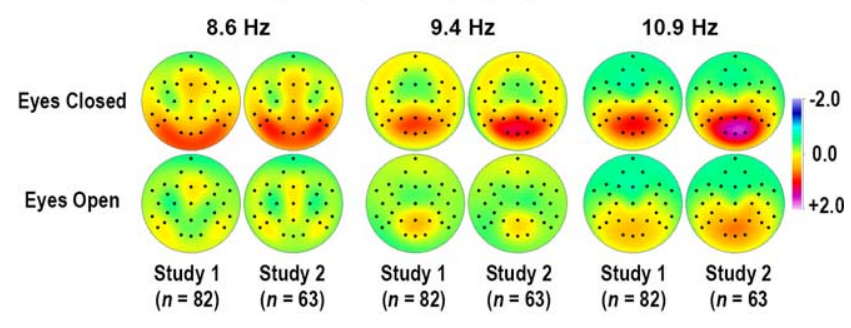

B Consistency of Alpha Topographies Across Groups

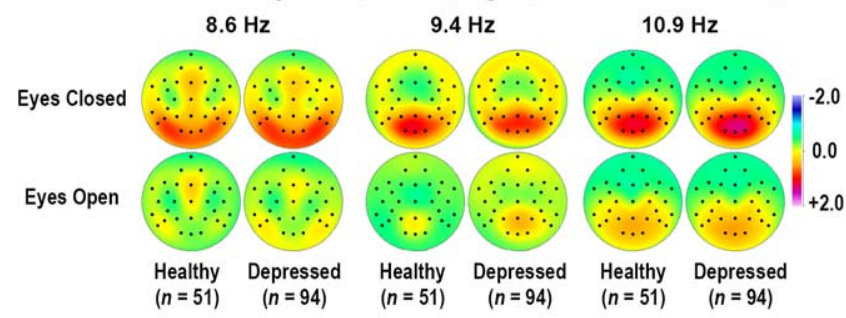

C Grouping By Factor Amplitude at Fz

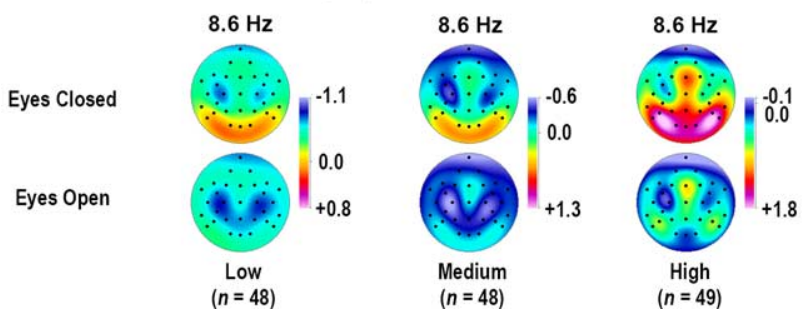

Fig. 4. Averaged topographies of the most prominent three alpha factors derived from CSD amplitude spectra for eyes-open and eyes-closed conditions. Topographies are shown for two separate studies (A), as well as for healthy controls and depressed patients averaged across the studies (B). Also shown are the mean topographies for three groups established by the amplitude of the 8.6- $\mathrm{Hz}$ factor at electrode $\mathrm{Fz}$ for eyes closed (C). These low, medium, and high groups differed in CSD factor amplitude not only at midline-frontal sites, but also at posterior sites; however, despite differences in amplitude, factor score topographies for eyes closed were similar for all three groups. Note that although scale resolution is the same, the absolute offset of each scale accommodates the group differences in amplitude.

is known to have a midline frontal topography. Likewise, it could be argued that the factor's inferior/posterior topography merely reflects the residual from a particularly broad posterior alpha topography. Moreover, no evidence has been shown to suggest that both topographic foci covary in amplitude. To evaluate the feasibility of such an association, participants were evenly divided into three groups based exclusively on the size of the $8.6-\mathrm{Hz}$ CSD amplitude spectrum factor at electrode $\mathrm{Fz}$ for eyes closed, and corresponding topographies for the high-, medium-, and low-Fz subjects were computed (ns were 49, 48, and 48, respectively). Despite marked quantitative differences in factor amplitude, all three groups showed similar topographies (Fig. 4C): (1) a primary posterior/inferior topography; (2) relatively greater amplitude at $\mathrm{Fz}$ than at 
other (i.e. not midline) frontocentral sites; (3) markedly greater amplitude for eyes-closed than eyes-open.

The association between the frontal and posterior foci suggests the possibility of an even stronger association: a direct linkage between the activity at the two widely separated sites. One possibility is that concurrent activity at the two sites may result from highly synchronized (coherent) processes, such as waveforms that are precisely in-phase $\left(0^{\circ}\right.$ : current sources or sinks at the same time in both regions), out of phase $\left(180^{\circ}\right.$ : sinks in one corresponding to sources in the other), or at another fixed phase angle. At the other extreme, it is possible that the signals at the two sites are phase independent (i.e. incoherent), but covary in amplitude alone. The likelihood of one or the other of these two extremes can have major implications for geometric, physiological, and statistical interpretations of the underlying neuronal generators.

Although an exhaustive description of these factors is beyond the scope of the present paper, it was reasoned that the most restrictive linkage between waveforms (i.e. constant phase angle) is necessary to suggest that both foci reflect similar patterns of spectral activity. To this end, we reviewed the epoched, filtered $(15 \mathrm{~Hz}$ low pass) CSD data for subjects showing the greatest factor amplitude at Fz, adding as a further restriction that rhythmic activity should be visible in individual CSD time epochs. Fig. 5A shows the characteristics of a representative CSD time epoch with these properties. The corresponding amplitude spectrum for this epoch was sharply-tuned at $8.6 \mathrm{~Hz}$, with a topography reminiscent of the corresponding fPCA factor (Fig. 5B). CSD alpha waveforms at midline frontal (Fz) and inferior/ posterior (P8) sites were closely time-locked and precisely out of phase throughout this epoch (i.e. sources at $\mathrm{Fz}$ correspond to sinks at $\mathrm{P} 8$, and visa versa). ${ }^{5}$ The wave-bywave topography of this linkage is also evident from sequential series of CSD maps corresponding to successive peaks (sinks, top series of maps) and troughs (sources, bottom series of maps) at Fz. ${ }^{6}$ A local Hjorth Laplacian produced the same result, indicating that the relationship was independent of the spherical model. Thus, a common alpha generator pattern can produce synchronized currents at frontal and inferior foci.

\section{Discussion}

This study evaluated the effectiveness of frequency PCA, which was performed on EEG amplitude, power, or logtransformed power spectra derived from nose-referenced

\footnotetext{
${ }^{5}$ It should be noted that for this strongly rhythmic epoch, the pairwise coherence spectrum for $\mathrm{Fz}$ versus all other electrodes was also maximal at P8, indicating a stable phase relationship between the two sites.

${ }^{6}$ An animated comparison of the corresponding EEG and CSD topographies during this epoch (i.e. for each time point) is posted at the following URL: http://psychophysiology.cpmc.columbia.edu/eegcsdepoch. html
}

surface potentials or their reference-free CSD transformations, to summarize and quantify EEG activity under the concept of a 'spectral component.' The suggested composite approach, combining PCA and CSD methodology using amplitude spectra, has a number of distinct advantages. First, the use of reference-free CSD waveforms preserves a direct relationship to the current generators that volume-conduct to produce the scalp-recorded EEG. As such, the neuroanatomical interpretations of regional findings stemming from these data are less ambiguous than their counterparts using conventional, reference-dependent EEG. Second, the subsequent use of an amplitude spectrum to transform and summarize CSD waveform topographies retains these linear properties, an advantage that is lost for conventional power or log power spectra. Third, instead of quantifying spectral components with rigid, a priori rectangular frequency bands, fPCA components adhere closely to the observed data, that is, their variance structure. Finally, fPCA efficiently identifies and removes artifact sources as linearly independent factors. Eye movement artifacts are confined to a low frequency component with a topography restricted to sites nearest the eyes, while muscle artifacts are identifiable by a high frequency spectrum and characteristic topography (Goncharova et al., 2003; Lee and Buchsbaum, 1987).

While none of the elements of the proposed method are new, even for EEG analyses, the suggested combination is unique and establishes an entirely new approach for identifying, measuring, and summarizing spectral EEG activity. Previously, for example, other investigators have recommended regression analysis (e.g. Davidson, 1988) or PCA (e.g. Wallstrom et al., 2004) for removing artifact from the resting EEG. Lagerlund et al. (2004) employed fPCA as an interactive filter to extract, and subsequently reconstruct, individual CSD time epochs from their complex Fourier spectra, using amplitude spectra for display. PCA has also been used to simplify the analysis of EEG power or log power for data restricted to fixed, predetermined frequency bands (Arruda et al., 1996). In contrast, the uniqueness of the present method originates from the exploitation of the complete spectral signature of EEG components and artifacts using one linear method without a priori limitation, yielding a completely 'data-driven' and at the same time physiologically-meaningful approach.

\subsection{Advantages of amplitude spectra}

Despite a priori reasons for focusing on fPCA solutions based on CSD amplitude spectra, the common use of log power spectra in quantitative EEG makes it of general interest to compare these solutions with those based on log power spectra, as well as the power spectra from which they are derived. The results showed that power spectrum fPCA solutions attenuated high frequencies and produced numerous alpha factors with overlapping or identical peak frequencies, thereby severely limiting their interpretation. On the other hand, log power spectra emphasized high 
A

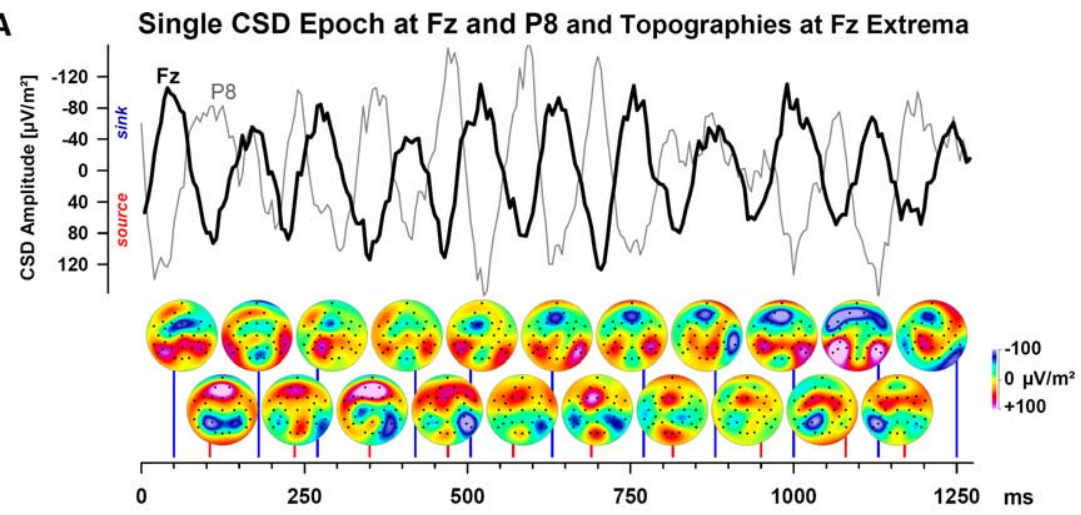

B

Amplitude Topographies at Discrete Frequencies Near Factor Peak

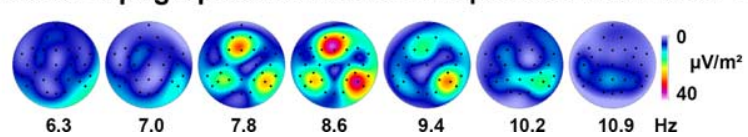

Fig. 5. (A) Representative eyes-closed CSD epoch for one participant with a high factor 8.6-Hz amplitude at Fz. Prominent CSD alpha rhythmicity at Fz and P8 tended to be precisely out of phase, with sources (sinks) at Fz being concurrent with sinks (sources) at P8. Corresponding CSD maps illustrate the linkage between waveforms at these sites (upper row refers to Fz sinks, bottom row refers to Fz sources). Fz sinks (cold colors at mid-frontal regions, blue lines refer to associated time point) are concurrent with inferior/posterior sources (warm colors), while Fz sources (warm colors at mid-frontal regions, red lines) frequently correspond to inferior/posterior sinks. Note that, unlike the waveform shown, all topographies were computed after smoothing the CSD by applying a 24-db, 15-Hz low pass filter. (B) The amplitude spectrum topography of the same CSD epoch shows spectral (maximum amplitude at $8.6 \mathrm{~Hz}$ ) and regional properties (maxima at midline frontal and inferior/posterior sites) consistent with a prominent 8.6-Hz CSD factor (cf. Fig. 4C).

frequencies (including line, CRT, and muscle artifacts) and produced few high-variance factors with broad spectral peaks. While the latter finding could imply that log power spectra are particularly helpful to quantify beta rather than alpha activity, researchers should be aware that highfrequency EEG topographies may easily be distorted by or result from a concurrent muscle artifact. As expected, amplitude spectra yielded solutions with the desirable outcome of an intermediate density (number and overlap of loadings) of alpha factors. In general, CSD fPCA solutions were similar to those derived from nosereferenced EEG spectra, but had the advantage of sharper factor score topographies, and produced factor loadings with less overlap, both in amplitude and spectral bandwidth, thereby increasing the uniqueness of each factor.

Across scaling methods, the topographies of EEG and CSD alpha factors varied systematically according to peak frequency, with low frequencies (theta/low alpha) including a midline frontocental and an inferior/posterior topography, while higher frequencies (mid and high alpha) showed the classic medial/posterior topography. CSD amplitude scaling produced the simplest fPCA solution, requiring only three spectrally- and topographically-distinct factors (i.e. minimal overlap) to account for most of the activity in the classic alpha band. Each of these alpha factors was present in two independent samples of healthy adults and depressed patients, and all were physiologically plausible (posterior topographies, greatest for eyes closed).

Furthermore, a comparison across scaling methods using fPCA indicates that the choice of a log power spectrum may misrepresent the underlying data, particularly when used in concert with predetermined frequency bands. Much of the variance about the mean of the EEG log power spectrum was summarized by a single alpha factor (additional alpha factors had lower variance) with a substantial roll-off into adjoining frequency bands (Fig. 3C), meaning that activity representing the traditional alpha band systematically covaries with theta and beta activity with this scaling. This finding indicates that an $8-13 \mathrm{~Hz}$ frequency band would be an arbitrary choice. Conversely, rectangular frequency estimates of alpha will also include contributions from spectral components outside the alpha band, most notably including EEG theta and muscle artifact, depending on the amount of overlap. This problem is particularly pervasive for regions and conditions in which alpha amplitudes are low (e.g. at frontal sites or at rest with eyes open).

\subsection{Limitations of the proposed method}

Despite the empirical and theoretical elegance of CSD methodology, a surface Laplacian estimate is restricted to the spatial domain in which the EEG is recorded: the scalp. Moreover, it disproportionately represents superficial generators (Nunez, 1990; Nunez et al., 1997; Srinivasan et al., 1998). Consequently, to the extent that EEG rhythms are widely synchronized across the scalp (Steriade, 2000), or are transmitted locally through cortex by wave-propagation (Robinson, 2003), local scalp-potential gradients may be sufficiently shallow to be poorly resolved by a surface 
Laplacian. In these cases, low-resolution estimates may actually give a better qualitative description of EEG generators than those observed using high resolution methods. When it is suspected that a CSD topography is over-resolved, this descriptive information may be restored by using a multiresolutional approach, either by varying the spatial filter properties of the CSD algorithm itself (e.g. using lower resolution subsets of electrodes; Tenke et al., 1993), or by exploiting the spatial filter properties of the different EEG measures (e.g. field potentials, CSD and cortical image; Nunez et al., 1997).

An additional concern is that CSD estimates are modeldependent, making it particularly important to assure that a chosen computational algorithm behaves appropriately for effects that have been determined to be important. However, algorithm- or model-dependent errors can be discounted if it can be shown that estimates based on different models produce similar solutions (e.g. Tandonnet et al., 2005, and Tenke et al., 1998, for similarity of local Hjorth and spherical spline Laplacians). Likewise, CSD estimates near the edges of the montage must always be interpreted with caution, since they are invariably based on insufficient sampling at these locations (i.e., there are no data for locations outside the montage).

Even without any evidence that a surface Laplacian is an effective method to summarize EEG spectra, the fact that it provides a reference-independent simplification of EEG topographies is by itself sufficient to endorse its use over traditional qEEG measures. Although it might be argued that more information is provided by other source imaging methods (for a review, see Michel et al., 2004), such as BESA (Scherg and von Cramon, 1985, 1986), which can precisely localize equivalent dipoles within a simplified geometric model, the solutions are non-unique and will fail if discrete, equivalent dipoles are inadequate to describe a particular generator. A useful alternative to fitting equivalent dipoles to the data is to localize the generators of Eq. (1) as smoothed, three-dimensional equivalent 'clouds' (LORETA; Pascual-Marqui et al., 1994). The most obvious shortcomings of this approach are that punctate generators will be smeared (Grave de Peralta Menendez and Gonzalez Andino, 2000), and the local sign (i.e. 'dipole orientation') of the generators may lose a precise relationship to regional cytoarchitecture (i.e. the degree of smoothing may be prevent an accurate representation of the pattern of intracortical sources and sinks, or their alignment with cortical projection cells). However, a greater concern for the present purposes is that the models themselves must be conducive to developing a quantitative method. In contrast to other methods, a surface Laplacian can be used as a preprocessing step that does not preclude either the production of spectral measures, or their subsequent simplification using fPCA.

In this study of resting EEG, conventional power spectrum averages were scaled to operationally define the 'effective' amplitude of a sinusoidal signal (Fourier component) by its RMS amplitude. Although power spectra are a methodological standard (e.g. Davidson et al., 2000; Pivik et al., 1993), its square root, the CSD amplitude spectrum recasts variance-related data in way that is more directly (and intuitively) related to the underlying volume-conductor model. Amplitude spectra thereby provide a closer analog to conventional peak or window measures of ERP amplitude, particularly when the EEG shows a stable rhythm. However, it cannot be inferred that amplitude scaling is optimal for active EEG paradigms, in which alpha is not necessarily the dominant physiological signal (e.g. Davidson et al., 1990; Koles et al., 2001; Lind et al., 1999; Papousek and Schulter, 2004). A recommendation for EEG measures in these tasks must await empirical evaluation.

Although the approach outlined here is considerably more powerful than similar methods in standard use, the amplitude spectrum is limited by its lack of phase information. For this reason, as outlined below, supplemental methods may be required to evaluate multiple generators. An alternative approach using cross spectra retains phase information (e.g. Lind et al., 1999; Pizzagalli et al., 2001). However, it should be noted that cross-spectrum means do not represent all activity, but rather all activity that is shared (correlated) across electrodes (e.g. Bendat and Piersol, 1971). The difference is not trivial, since the two approaches summarize a fundamentally distinct subset of activity from that described by either CSD amplitude spectra or conventional EEG power spectra.

The use of fPCA methodology completely avoids the problems inherent in the use of rigidly-defined rectangular frequency bands by matching non-rectangular templates (i.e. components) to the variance structure of the data. Following the approach developed for ERPs (Kayser and Tenke, 2003), we used a covariance-based PCA with unrestricted factor extraction and Varimax rotation of the unscaled (covariance) loadings. Using this approach, ERP factors were more directly interpretable (i.e. more useful) when extracted using a covariance rather than correlation association matrix, and the components were better defined and more stable using unrestricted solutions rather than solutions employing factor retention criteria (e.g. a Scree test). High-variance factors tended to improve rapidly as successive factors were extracted, reflecting, at least in part, the removal of noise from overlapping waveforms of interest. However, lowvariance factors required cautious consideration, and were regarded as interpretable when, and only when, the shape of the loadings and the topography of the factor scores were both consistent with an independently-verified physiological component (Kayser and Tenke, 2003). These cautions apply even more strongly to fPCA, since the properties of spectral waveforms are not as well understood. However, it was previously shown that the general principles of an unrestricted, covariance-based Varimax-PCA detailed for ERP data (Kayser and Tenke, 2003) also apply to nose-referenced EEG spectra (Kayser et al., 2000). 
For the temporal PCA used with ERP data, the prestimulus period provides appreciable information about the quality of a baseline-corrected ERP recording, as well as about the interpretability of a PCA factor solution (Kayser and Tenke, 2003). The variance is small near the onset of the stimulus, and large near the end of the recording epoch, and at treatment-dependent ERP component peaks (Kayser and Tenke, 2003; Lehmann and Skrandies, 1980; van Boxtel, 1998). For spectral data, the highest signal variance is generally observed at the lowest frequencies (i.e. the beginning of the spectral epoch), while an identifiable 'zero' at high frequencies can serve a comparable function for fPCA solutions. Since large amplitude loadings at high frequencies are likely to represent or include artifact, such factors should be interpreted only after a critical evaluation of factor score topographies. As with ERP data, negativevalued regions of a fPCA topography must also be interpreted with caution, as the sign of the factor scores reflect variations around the grand mean that has been removed by factoring the covariance matrix. Since amplitude spectra are exclusively positive-valued, the numerical zero used as a reference point has a different meaning than it does for a temporal PCA derived from baseline-corrected ERP data. ${ }^{7}$ As a final limitation, the impact of additional parametric variations should also be explored (e.g. epoch duration, which is inversely related to the frequency resolution of the FFT), as the present study conveniently applied PCA methodology optimized for ERP data (Kayser and Tenke, 2003).

\subsection{Identification of spectral components of the EEG}

The topography and symmetry of EEG alpha figures prominently in recent models of brain function and psychopathology, with a particular emphasis on frontal alpha (Bruder et al., 1997; Davidson, 1998; Hagemann, 2004; Heller et al., 1995; Shankman et al., 2005). However, the stability and asymmetry of the alpha topography may vary, depending on the electrode site and the recording reference (cf. Fig. 1; Allen et al., 2004a, b; Debener et al., 2000a; Hagemann et al., 2001; Reid et al., 1998). Inasmuch as the CSD fPCA detailed here offers a reliable, reference-independent approach that summarizes the distribution of the underlying current generators, it could provide a much-needed standard for this difficult field.

Alpha rhythmicity in the resting EEG has a welldocumented posterior topography. The fPCA solutions all support this classic property for high-frequency alpha (peaks $>10 \mathrm{~Hz}$ ). However, additional low-frequency alpha

\footnotetext{
${ }^{7}$ All values of the grand mean amplitude spectra waveform are positivevalued (i.e. equal to or greater than zero). In contrast, the values of a grand mean ERP waveform can have either sign. In both cases, however, the sign of the factor scores must be interpreted with respect to the corresponding loading interval of the grand mean waveform for a given factor.
}

factors were also extracted in most solutions, characterized by inferior/posterior and midline anterior topographies. Although fPCA solutions for EEG and CSD differed in the number and prominence of alpha factors, the solution for reference-free CSD amplitude spectra was the most simple, separating a low alpha factor from a high alpha factor, as well as an additional intermediate alpha factor. The latter alpha factor had a sharper and more symmetric loading waveform than the other alpha factors, with a midline posterior topography that was similar to, but shallower than that of the high-alpha factor. In contrast, low alpha was maximal at inferior locations. By virtue of the CSD transformation, it can be concluded that the generators of all factors were localizable to posterior brain regions, which is consistent with previous surface Laplacian descriptions (Nunez et al., 1997; Srinivasan et al., 1998). In further accordance with the concept of a spectral component, all three alpha factors showed a condition-related dependency (i.e. greater amplitude with eyes closed), and were reproducible across groups and studies. Rhythmicity in motor regions attributable to mu rhythm was not observed among the highvariance factors (i.e. the first 10 extracted) in our sample of resting EEG, suggesting that this phenomenon does not account for or contribute to the alpha topographies observed on a group level. Similarly, and notably, no factor suggested generators on the dorsal surface of the frontal lobes. The lack of evidence for additional frontal generators in such a large sample is problematical for existing models of resting EEG asymmetries.

The rising slope of the low alpha loading spectrum includes a prominent theta contribution, with a topography that includes a secondary focus on the frontal midline. Interestingly, task-dependent theta has a similar midlinefrontal topography (Gevins and Smith, 2000; Gevins et al., 1997; McEvoy et al., 2001). By submitting crossspectra (i.e. unscaled coherent activity) to LORETA, Pizzagalli et al. (2001) localized the effective generators of task-related theta to anterior cingulate cortex. Luu et al. (2004) found evidence for a midfrontal theta contribution to the errorrelated negativity. Using Parallel Factor Analysis and PCA, Miwakeichi et al. (2004) reported a comparable localization of scalp- and intracranially-recorded theta during arithmetic tasks. Moreover, theta during working memory tasks is synchronized between prefrontal and posterior association regions (Sarnthein et al., 1998), and can be recorded from extrastriate visual cortex (monkeys; Lee et al., 2005). Although caution must be used when comparing the spectral properties of the resting and task-related EEG (as well as ERP), it is tempting to suggest a common anatomical and physiological origin (i.e. the 'low alpha' factor reported here represents activity in the theta band), particularly considering the report of an anterior-midline, lorazepam-resistant, resting EEG alpha (LORETA; Connemann et al., 2005).

Although the scalp-recorded EEG represents volumeconducted activity, local intracranial cancellation (i.e. partial field closure) can have a measurable impact on a topography. Such properties may take the form of abrupt 
transitions or discontinuities in an otherwise regular topography. One model-dependent implication of these irregularities is the occasional localization of a physiologically implausible generator, such as a radially-oriented dipole aligned within the frontal midline. A physiologically plausible generator must represent both the location and the organization of the tissue, so that the dipole-equivalent to a laminar pattern of sources and sinks must be oriented normal to the cortical surface ('cortical dipole' of Lorente de No, 1947; also see Mitzdorf, 1985; Tenke et al., 1993). However, bilateral activation of homologous regions in both hemispheres must be resolved as a pair of tangentiallyoriented dipoles in direct opposition to each, reflecting a local field that is largely, but incompletely, cancelled, to leave a small, sharply-localized residual at the midline (cf. Fig. 3 of Tenke et al., 1993).

The secondary midline frontal focus illustrates both the strengths and the limitations of the composite fPCA approach based on CSD amplitude spectra. The sharplylocalized topography along the frontal midline is consistent with an effective midline generator, resulting from the synchronized, bilateral activation of symmetric cortical regions on both surfaces within the longitudinal fissure. However, a CSD topography produced by bilateral generators confined to the walls of the fissure would either be displaced to the lateral surface of the frontal lobes (analogous to the displacement of auditory N1 CSD topography from the Sylvian fissue; cf. Fig. 2 of Tenke et al., 1998), or else would produce field closure artifacts at nearby electrodes (e.g. sinks at Fz surrounded by smaller sources at F3 and F4; Tenke et al., 1993, and unpublished observations of surface Laplacian topographies for simulated closed fields). These distinctive properties should be clearest in individual cases and records, where there is no possibility of smearing across subjects or states.

The representative example given in Fig. 5 argues strongly against a bilateral, sulcal generator based on two observations: (1) CSD sources and sinks were not strictly confined to midline electrodes; (2) midline sources and sinks were not coupled with misallocated complements (i.e. sinks and sources, respectively) at midfrontal sites (i.e. F3/4). Moreover, a deeper pair of sulcal generators (e.g. bilateral cingulate cortex), if their activity could indeed be recorded at scalp, is more likely to produce a bilateral CSD topography that is displaced from the midline. This is due both to the extensive cancellation of the opposing effective dipoles at a (tangential) distance and to the reinforcement of the field potential orthogonal to the lamination of the cortex (i.e. in the direction of the underlying white matter). Given these considerations, we conclude that the high-amplitude,

\footnotetext{
${ }^{8}$ A precise model of the properties of putative midline generators could only be produced from the laminar pattern of CSD sources and sinks obtained from intracranial recordings.
}

midline-frontal alpha observed here originated from generators distributed over cortex within and adjacent to the longitudinal fissure (i.e. near the convexity), rather than from deep within the fissure (e.g. cingulate cortex). ${ }^{8}$

It may be argued that the fPCA of the CSD amplitude spectrum provided an oversimplified solution, statistically combining the contributions of inferior/posterior low alpha with an independent midline theta that has a similar spectrum. Although we cannot universally refute the possibility, the present findings do indicate that the activity of the midline-frontal generator is associated with the activity at posterior/inferior sites. The prominence of the frontal focus varies across subjects in direct relation to the amplitude of the larger, posterior alpha generator (Fig. 4C). Consistently, the sample epoch illustrated in Fig. 5 indicates a precise inversion of frontal midline and posterior/inferior CSD waveforms. This pattern is not a result of the spherical Laplacian model, because the same relationship was verified using a planar local Hjorth Laplacian. The close association between regions was further supported by examining pairwise coherences with $\mathrm{Fz}$ between electrodes, which were similar to the power spectrum shown in Fig. 5.

Finally, one could suggest that a single pair of posterior generators can account for the entire topography. Specifically, frontal alpha activity may merely reflect the intersection of the underside of posterior dipoles. However, this degree of symmetry and focus is unlikely for a CSD feature recorded at such a distance from its underlying generator. Moreover, a single pair of posterior generators cannot account for all cases, most notably the pattern illustrated in Fig. 5, because midline and posterior CSD amplitudes are comparable, and posterior activity is asymmetric. Therefore, we conclude that the midline frontal CSD focus reflects a distinct neuronal generator that may, at times, synchronize with activity at posterior sites.

\section{Acknowledgements}

This work was supported in part by grant MH36295 from the National Institute of Mental Health (NIMH).

We greatly appreciate the assistance of Nil Bhattacharya, Carlye Griggs, Paul Leite, Mia Sage, Stewart Shankman, and Barbara Stuart with data collection, storage, and preprocessing.

Waveform plotting software was written by Charles L. Brown, III, who also provided helpful insights during the implementation of the spherical spline algorithm, benefitting from gracious advice given earlier by Patrick Berg.

A preliminary summary of this report has been presented at the 43rd Annual Meeting of the Society for Psychophysiological Research (SPR), October 2003, Chicago, IL. 


\section{References}

Achim A, Marcantoni W. Principal component analysis of event-related potentials: misallocation of variance revisited. Psychophysiology 1997; 34(5):597-606.

Allen JJ, Kline JP. Frontal EEG asymmetry, emotion, and psychopathology: the first, and the next 25 years. Biol Psychol 2004; 67(1-2):1-5.

Allen JJ, Coan JA, Nazarian M. Issues and assumptions on the road from raw signals to metrics of frontal EEG asymmetry in emotion. Biol Psychol 2004a;67(1-2):183-218.

Allen JJ, Urry HL, Hitt SK, Coan JA. The stability of resting frontal electroencephalographic asymmetry in depression. Psychophysiology 2004b;41(2):269-80.

Andresen B. Multivariate statistical methods and their capability to demarcate psychophysiologically and neurophysiologically sound frequency components of human scalp EEG. In: Zschocke S, Speckmann E-J, editors. Basic mechanisms of the EEG. Boston, MT: Birkhauser; 1993. p. 317-52.

Andresen B, Stemmler G, Thom E, Irrgang E. Methodological conditions of congruent factors-a comparison of EEG frequency structure between hemispheres. Multivar Behav Res 1984;19(1):3-32.

Arruda JE, Weiler MD, Valentino D, Willis WG, Rossi JS, Stern RA, Gold SM, Costa L. A guide for applying principal-components analysis and confirmatory factor analysis to quantitative electroencephalogram data. Int J Psychophysiol 1996;23(1-2):63-81.

Babiloni F, Cincotti F, Bianchi L, Pirri G, del R Millan J, Mourino J, Salinari S, Marciani MG. Recognition of imagined hand movements with low resolution surface Laplacian and linear classifiers. Med Eng Phys 2001;23(5):323-8.

Babiloni C, Babiloni F, Carducci F, Cincotti F, Rosciarelli F, Arendt Nielsen L, Chen AC, Rossini PM. Human brain oscillatory activity phase-locked to painful electrical stimulations: a multi-channel EEG study. Hum Brain Mapp 2002;15(2):112-23.

Babiloni C, Babiloni F, Carducci F, Cappa SF, Cincotti F, Del Percio C, Miniussi C, Moretti DV, Rossi S, Sosta K, Rossini PM. Human cortical responses during one-bit short-term memory. A high-resolution EEG study on delayed choice reaction time tasks. Clin Neurophysiol 2004; 115(1):161-70.

Basar E. Towards a renaissance of 'alphas'. Int J Psychophysiol 1997; 26(1-3):1-3.

Beauducel A, Debener S. Misallocation of variance in event-related potentials: simulation studies on the effects of test power, topography, and baseline-to-peak versus principal component quantifications. J Neurosci Methods 2003;124(1):103-12.

Bendat JS, Piersol AG. Random data: analysis and measurement procedures. New York, NY: Wiley-Interscience; 1971.

Bruder GE, Fong R, Tenke CE, Leite P, Towey JP, Stewart JE, McGrath PJ, Quitkin FM. Regional brain asymmetries in major depression with or without an anxiety disorder: a quantitative electroencephalographic study. Biol Psychiatry 1997;41(9):939-48.

Bruder GE, Kayser J, Tenke CE, Leite P, Schneier FR, Stewart JW, Quitkin FM. Cognitive ERPs in depressive and anxiety disorders during tonal and phonetic oddball tasks. Clin Electroencephalogr 2002;33(3): $119-24$.

Burgess AP, Gruzelier J. How reproducible is the topographical distribution of EEG amplitude? Int J Psychophysiol 1997;26(1-3):113-9.

Buzsaki G. The thalamic clock: emergent network properties. Neuroscience 1991;41(2-3):351-64.

Buzsaki G, Czopf J, Kondakor I, Kellenyi L. Laminar distribution of hippocampal rhythmic slow activity (RSA) in the behaving rat: currentsource density analysis, effects of urethane and atropine. Brain Res 1986;365(1):125-37.

Cattell RB. Scree test for number of factors. Multivar Behav Res 1966;1(2): 245-76.
Chapman RM, McCrary JW. EP component identification and measurement by principal components analysis. Brain Cogn 1995;27(3): 288-310.

Cincotti F, Mattia D, Babiloni C, Carducci F, Salinari S, Bianchi L, Marciani MG, Babiloni F. The use of EEG modifications due to motor imagery for brain-computer interfaces. IEEE Trans Neural Syst Rehabil Eng 2003;11(2):131-3.

Connemann BJ, Mann K, Lange Asschenfeldt C, Ruchsow M, Schreckenberger M, Bartenstein P, Grunder G. Anterior limbic alphalike activity: a low resolution electromagnetic tomography study with lorazepam challenge. Clin Neurophysiol 2005;116(4):886-94.

Cook IA, O'Hara R, Uijtdehaage SH, Mandelkern M, Leuchter AF. Assessing the accuracy of topographic EEG mapping for determining local brain function. Electroencephalogr Clin Neurophysiol 1998; 107(6):408-14.

Davidson RJ. EEG measures of cerebral asymmetry: conceptual and methodological issues. Int J Neurosci 1988;39(1-2):71-89.

Davidson RJ. Affective style and affective disorders: perspectives from affective neuroscience. Cognition Emotion 1998;12(3):307-30.

Davidson RJ, Fox NA. Frontal brain asymmetry predicts infants' response to maternal separation. J Abnorm Psychol 1989;98(2):127-31.

Davidson RJ, Chapman JP, Chapman LJ, Henriques JB. Asymmetrical brain electrical activity discriminates between psychometricallymatched verbal and spatial cognitive tasks. Psychophysiology 1990; 27(5):528-43.

Davidson RJ, Jackson DC, Larson CL. Human electroencephalography. In: Cacioppo JT, Tassinary LG, Bernston GG, editors. Handbook of psychophysiology. 2nd ed. Cambridge: Cambridge University Press; 2000. p. 27-52.

Debener S, Beauducel A, Brocke B, Kayser J. Resting anterior EEG alpha asymmetry and affective style: effects of electrode location and reference. J Psychophysiol 2000a;14(1):62.

Debener S, Kayser J, Tenke CE, Beauducel A. Principal components analysis (PCA) as a tool for identifying EEG frequency bands: II. Dissociation of resting alpha asymmetries. Psychophysiology 2000b; 37:S35.

Dien J. Addressing misallocation of variance in principal components analysis of event-related potentials. Brain Topogr 1998;11(1):43-55.

Dixon WJ, editor. BMDP statistical software manual: to accompany the 7.0 software release. Berkeley, CA: University of California Press; 1992.

Donchin E. A multivariate approach to the analysis of average evoked potentials. IEEE Trans Biomed Eng 1966;13(3):131-9.

Donchin E, Heffley EF. Multivariate analysis of event-related potential data: a tutorial review. In: Otto DA, editor. Multidisciplinary perspectives in event-related brain potential research. Proceedings of the fourth international congress on event-related slow potentials of the brain (EPIC IV), Hendersonville, NC, April 4-10, 1976. Washington, DC: The Office; 1978. p. 555-72.

Duffy FH, Bartels PH, Burchfiel JL. Significance probability mapping: an aid in the topographic analysis of brain electrical activity. Electroencephalogr Clin Neurophysiol 1981;51(5):455-62.

Duffy FH, Hughes JR, Miranda F, Bernad P, Cook P. Status of quantitative EEG (QEEG) in clinical practice. Clin Electroencephalogr 1994;25(4): VI-XXII.

Gasser T, Molinari L. The analysis of the EEG. Stat Methods Med Res 1996;5(1):67-99.

Gevins A, Smith ME. Neurophysiological measures of working memory and individual differences in cognitive ability and cognitive style. Cereb Cortex 2000;10(9):829-39.

Gevins A, Smith ME, McEvoy L, Yu D. High-resolution EEG mapping of cortical activation related to working memory: effects of task difficulty, type of processing, and practice. Cereb Cortex 1997;7(4):374-85.

Glaser EM, Ruchkin DS. Principles of neurobiological signal analysis. New York: Academic Press; 1976.

Gloor P. Hans Berger and the discovery of the electroencephalogram. Electroencephalogr Clin Neurophysiol Suppl 1969;28:1-36. 
Goncharova II, McFarland DJ, Vaughan TM, Wolpaw JR. EMG contamination of EEG: spectral and topographical characteristics. Clin Neurophysiol 2003;114(9):1580-93.

Gratton G, Coles MGH, Donchin E. A new method for off-line removal of ocular artifact. Electroencephalogr Clin Neurophysiol 1983;55(4): 468-84.

Grave de Peralta Menendez R, Gonzalez Andino SL. Discussing the capabilities of Laplacian minimization. Brain Topogr 2000;13(2): 97-104.

Hagemann D. Individual differences in anterior EEG asymmetry: methodological problems and solutions. Biol Psychol 2004;67(1-2): 157-82.

Hagemann D, Naumann E, Thayer JF. The quest for the EEG reference revisited: a glance from brain asymmetry research. Psychophysiology 2001;38(5):847-57.

Heller W, Etienne MA, Miller GA. Patterns of perceptual asymmetry in depression and anxiety: implications for neuropsychological models of emotion and psychopathology. J Abnorm Psychol 1995;104(2):327-33.

Henriques JB, Davidson RJ. Regional brain electrical asymmetries discriminate between previously depressed and healthy control subjects. J Abnorm Psychol 1990;99(1):22-31.

Henriques JB, Davidson RJ. Brain electrical asymmetries during cognitive task performance in depressed and nondepressed subjects. Biol Psychiatry 1997;42(11):1039-50.

Hjorth B. An on-line transformation of EEG scalp potentials into orthogonal source derivations. Electroencephalogr Clin Neurophysiol Suppl 1975;39(5):526-30.

Holsheimer J. Electrical conductivity of the hippocampal CA1 layers and application to current-source-density analysis. Exp Brain Res 1987; 67(2):402-10.

Horn JL. A rationale and test for the number of factors in factor-analysis. Psychometrika 1965;30(2):179-85.

Ishii R, Dziewas R, Chau W, Soros P, Okamoto H, Gunji A, Pantev C. Current source density distribution of sleep spindles in humans as found by synthetic aperture magnetometry. Neurosci Lett 2003;340(1):25-8.

Junghöfer M, Elbert T, Tucker DM, Braun C. The polar average reference effect: a bias in estimating the head surface integral in EEG recording. Clin Neurophysiol 1999;110(6):1149-55.

Kaiser HF. The application of electronic computers to factor analysis. Educ Psychol Meas 1960;20:141-51.

Kayser J, Tenke CE. Optimizing PCA methodology for ERP component identification and measurement: theoretical rationale and empirical evaluation. Clin Neurophysiol 2003;114(12):2307-25.

Kayser J, Tenke CE. Trusting in or breaking with convention: towards a renaissance of principal components analysis in electrophysiology. Clin Neurophysiol 2005;116(8):1747-53.

Kayser J, Tenke CE. Principal components analysis of Laplacian waveforms as a generic method for identifying ERP generator patterns: I. Evaluation with auditory oddball tasks. Clin Neurophysiol, in press a.

Kayser J, Tenke CE. Principal components analysis of Laplacian waveforms as a generic method for identifying ERP generator patterns: II. Adequacy of low-density estimates. Clin Neurophysiol, in press b.

Kayser J, Tenke CE, Debener S. Principal components analysis (PCA) as a tool for identifying EEG frequency bands: I. Methodological considerations and preliminary findings. Psychophysiology 2000;37: S54.

Koles ZJ, Flor Henry P, Lind JC. Low-resolution electrical tomography of the brain during psychometrically matched verbal and spatial cognitive tasks. Hum Brain Mapp 2001;12(3):144-56.

Kraut MA, Arezzo JC, Vaughan Jr HG. Intracortical generators of the flash VEP in monkeys. Electroencephalogr Clin Neurophysiol 1985;62(4): 300-12.

Lagerlund TD, Sharbrough FW, Busacker NE. Use of principal component analysis in the frequency domain for mapping electroencephalographic activities: comparison with phase-encoded Fourier spectral analysis. Brain Topogr 2004;17(2):73-84.
Lee S, Buchsbaum MS. Topographic mapping of EEG artifacts. Clin Electroencephalogr 1987;18(2):61-7.

Lee H, Simpson GV, Logothetis NK, Rainer G. Phase locking of single neuron activity to theta oscillations during working memory in monkey extrastriate visual cortex. Neuron 2005;45:147-56.

Lehmann D, Skrandies W. Reference-free identification of components of checkerboard-evoked multichannel potential fields. Electroencephalogr Clin Neurophysiol 1980;48(6):609-21.

Leuchter AF, Cook IA, Lufkin RB, Dunkin J, Newton TF, Cummings JL, Mackey JK, Walter DO. Cordance: a new method for assessment of cerebral perfusion and metabolism using quantitative electroencephalography. Neuroimage 1994;1(3):208-19.

Lind JC, Flor Henry P, Koles ZJ. Discriminant analysis and equivalent source localization of the EEG related to cognitive functions. Brain Topogr 1999;11(4):265-78.

Logothetis NK. The underpinnings of the BOLD functional magnetic resonance imaging signal. J Neurosci 2003;23(10):3963-71.

Logothetis NK, Pauls J, Augath M, Trinath T, Oeltermann A. Neurophysiological investigation of the basis of the fMRI signal. Nature 2001; 412(6843):150-7.

Lopes da Silva F. Neural mechanisms underlying brain waves: from neural membranes to networks. Electroencephalogr Clin Neurophysiol 1991; 79(2):81-93.

Lorente de No RA. A study of nerve physiology (Rockefeller Institute for Medical Research, study 132). New York: Rockefeller Institute; 1947 p. 389-477.

Luu P, Tucker DM, Makeig S. Frontal midline theta and the error-related negativity: neurophysiological mechanisms of action regulation. Clin Neurophysiol 2004;115(8):1821-35.

McEvoy LK, Pellouchoud E, Smith ME, Gevins A. Neurophysiological signals of working memory in normal aging. Brain Res Cogn Brain Res 2001;11(3):363-76.

Michel CM, Murray MM, Lantz G, Gonzalez S, Spinelli L, Grave de Peralta R. EEG source imaging. Clin Neurophysiol 2004;115(10): 2195-222.

Mitzdorf U. Current source-density method and application in cat cerebral cortex: investigation of evoked potentials and EEG phenomena. Physiol Rev 1985;65(1):37-100.

Miwakeichi F, Martinez Montes E, Valdes Sosa PA, Nishiyama N, Mizuhara H, Yamaguchi Y. Decomposing EEG data into space-timefrequency components using parallel factor analysis. Neuroimage 2004; 22(3):1035-45.

Möcks J, Verleger R. Principal component analysis of event-related potentials: a note on misallocation of variance. Electroencephalogr Clin Neurophysiol 1986;65(5):393-8.

Näätänen R, Picton TW. The N1 wave of the human electric and magnetic response to sound: a review and an analysis of the component structure. Psychophysiology 1987;24(4):375-425.

Nicholson C. Theoretical analysis of field potentials in anisotropic ensembles of neuronal elements. IEEE Trans Biomed Eng 1973; 20(4):278-88.

Nicholson C, Freeman JA. Theory of current source-density analysis and determination of conductivity tensor for anuran cerebellum. J Neurophysiol 1975;38(2):356-68.

Niedermeyer E. The normal EEG in the waking adult. In: Niedermeyer E, Lopes da Silva F, editors. Electroencephalography: basic principles, clinical applications and related fields. 2nd ed. Baltimore, MD: Urban \& Schwarzenberg; 1987. p. 97-117.

Niedermeyer E. Alpha rhythms as physiological and abnormal phenomena. Int J Psychophysiol 1997;26(1-3):31-49.

Nunez PL. Localization of brain activity with electroencephalography. Adv Neurol 1990;54:39-65.

Nunez PL, Silberstein RB, Cadusch PJ, Wijesinghe RS, Westdorp AF, Srinivasan R. A theroretical and experimental study of high resolution EEG based on surface Laplacians and cortical imaging. Electroencephalogr Clin Neurophysiol 1994;90:40-57. 
Nunez PL, Srinivasan R, Westdorp AF, Wijesinghe RS, Tucker DM, Silberstein RB, Cadusch PJ. EEG coherency. I: statistics, reference electrode, volume conduction, Laplacians, cortical imaging, and interpretation at multiple scales. Electroencephalogr Clin Neurophysiol 1997;103(5):499-515.

Oldfield RC. The assessment and analysis of handedness: the Edinburgh inventory. Neuropsychologia 1971;9(1):97-113.

Oostendorp TF, van Oosterom A. The surface Laplacian of the potential: theory and application. IEEE Trans Biomed Eng 1996;43(4):394-405.

Papousek I, Schulter G. Manipulation of frontal brain asymmetry by cognitive tasks. Brain Cogn 2004;54(1):43-51.

Pascual-Marqui RD, Michel CM, Lehmann D. Low resolution electromagnetic tomography: a new method for localizing electrical activity in the brain. Int J Psychophysiol 1994;18(1):49-65.

Pernier J, Perrin F, Bertrand O. Scalp current density fields: concept and properties. Electroencephalogr Clin Neurophysiol 1988;69(4):385-9.

Perrin F, Pernier J, Bertrand O, Echallier JF. Spherical splines for scalp potential and current density mapping. Electroencephalogr Clin Neurophysiol 1989;72(2):184-7 [Corrigenda EEG 02274, Clin Neurophysiol, 1990, 76, 565].

Perrin F, Pernier J, Bertrand O, Echallier JF. Corrigenda EEG 02274. Electroencephalogr Clin Neurophysiol 1990;76:565.

Pfurtscheller G. Induced oscillations in the alpha band: functional meaning. Epilepsia 2003;4412(Suppl. 12):2-8.

Picton TW, Bentin S, Berg P, Donchin E, Hillyard SA, Johnson Jr R, Miller GA, Ritter W, Ruchkin DS, Rugg MD, Taylor MJ. Guidelines for using human event-related potentials to study cognition: recording standards and publication criteria. Psychophysiology 2000;37(2): 127-52.

Pineda JA, Silverman DS, Vankov A, Hestenes J. Learning to control brain rhythms: making a brain-computer interface possible. IEEE Trans Neural Syst Rehabil Eng 2003;11(2):181-4.

Pivik RT, Broughton RJ, Coppola R, Davidson RJ, Fox N, Nuwer MR. Guidelines for the recording and quantitative analysis of electroencephalographic activity in research contexts. Psychophysiology 1993;30(6):547-58.

Pizzagalli D, Pascual Marqui RD, Nitschke JB, Oakes TR, Larson CL, Abercrombie HC, Schaefer SM, Koger JV, Benca RM, Davidson RJ. Anterior cingulate activity as a predictor of degree of treatment response in major depression: evidence from brain electrical tomography analysis. Am J Psychiatry 2001;158(3):405-15.

Pollock VE, Schneider LS, Lyness SA. Reliability of topographic quantitative EEG amplitude in healthy late-middle-aged and elderly subjects. Electroencephalogr Clin Neurophysiol 1991;79(1):20-6.

Reid SA, Duke LM, Allen JJ. Resting frontal electroencephalographic asymmetry in depression: inconsistencies suggest the need to identify mediating factors. Psychophysiology 1998;35(4):389-404.

Robinson PA. Neurophysical theory of coherence and correlations of electroencephalographic and electrocorticographic signals. J Theor Biol 2003;222(2):163-75.

Sarnthein J, Petsche H, Rappelsberger P, Shaw GL, von Stein A. Synchronization between prefrontal and posterior association cortex during human working memory. Proc Natl Acad Sci USA 1998;95: 7092-6.

Scherg M, von Cramon D. Two bilateral sources of the late AEP as identified by a spatio-temporal dipole model. Electroencephalogr Clin Neurophysiol 1985;62(1):32-44.

Scherg M, von Cramon D. Evoked dipole source potentials of the human auditory cortex. Electroencephalogr Clin Neurophysiol 1986;65(5): 344-60.

Schroeder CE, Tenke CE, Givre SJ. Subcortical contributions to the surface-recorded flash-VEP in the awake macaque. Electroencephalogr Clin Neurophysiol 1992;84:219-31.
Semlitsch HV, Anderer P, Schuster P, Presslich O. A solution for reliable and valid reduction of ocular artifacts, applied to the P300 ERP. Psychophysiology 1986;23(6):695-703.

Shankman SA, Tenke CE, Bruder GE, Durbin CE, Hayden EP, Klein DN. Low positive emotionality and EEG asymmetry. Dev Psychopathol 2005;17:85-98.

Simson R, Vaughan Jr HG, Ritter W. The scalp topography of potentials associated with missing visual or auditory stimuli. Electroencephalogr Clin Neurophysiol 1976;40(1):33-42.

Spencer KM, Dien J, Donchin E. A componential analysis of the ERP elicited by novel events using a dense electrode array. Psychophysiology 1999;36(3):409-14.

Spencer KM, Dien J, Donchin E. Spatiotemporal analysis of the late ERP responses to deviant stimuli. Psychophysiology 2001;38(2):343-58.

Srinivasan R, Nunez PL, Silberstein RB. Spatial filtering and neocortical dynamics: estimates of EEG coherence. IEEE Trans Biomed Eng 1998; 45(7):814-26.

Steriade M. Corticothalamic resonance, states of vigilance and mentation. Neuroscience 2000;101(2):243-76.

Steriade M, McCormick DA, Sejnowski TJ. Thalamocortical oscillations in the sleeping and aroused brain. Science 1993;262(5134):679-85.

Tandonnet C, Burle B, Hasbroucq T, Vidal F. Spatial enhancement of EEG traces by surface Laplacian estimation: comparison between local and global methods. Clin Neurophysiol 2005;116(1):18-24.

Tenke CE. Statistical characterization of the EEG: the use of the power spectrum as a measure of ergodicity. Electroencephalogr Clin Neurophysiol 1986;63(5):488-93.

Tenke CE, Kayser J. A convenient method for detecting electrolyte bridges in multichannel electroencephalogram and event-related potential recordings. Clin Neurophysiol 2001;112(3):545-50.

Tenke CE, Kayser J, Fong R, Leite P, Towey JP, Bruder GE. Response- and stimulus-related ERP asymmetries in a tonal oddball task: a Laplacian analysis. Brain Topogr 1998;10(3):201-10.

Tenke CE, Schroeder CE, Arezzo JC, Vaughan Jr HG. Interpretation of high-resolution current source density profiles: a simulation of sublaminar contributions to the visual evoked potential. Exp Brain Res 1993;94(2):183-92.

Timofeev I, Grenier F, Bazhenov M, Houweling AR, Sejnowski TJ, Steriade M. Short- and medium-term plasticity associated with augmenting responses in cortical slabs and spindles in intact cortex of cats in vivo. J Physiol 2002;542(2):583-98.

van Boxtel GJM. Computational and statistical methods for analyzing event-related potential data. Behav Res Methods Instrum Comput 1998; 30(1):87-102.

Wallstrom GL, Kass RE, Miller A, Cohn JF, Fox NA. Automatic correction of ocular artifacts in the EEG: a comparison of regression-based and component-based methods. Int J Psychophysiol 2004;53(2):105-19.

Woestenburg JC, Verbaten MN, Slangen JL. Stimulus information and habituation of the visual event related potential and the skin conductance reaction under task-relevance conditions. Biol Psychol 1983; 16:225-40.

Wolpaw JR, McFarland DJ. Multichannel EEG-based brain-computer communication. Electroencephalogr Clin Neurophysiol 1994;90(6): 444-9.

Wood CC, McCarthy G. Principal component analysis of event-related potentials: simulation studies demonstrate misallocation of variance across components.. Electroencephalogr Clin Neurophysiol 1984;59(3): 249-60.

Yao D. The theoretical relation of scalp Laplacian and scalp current density of a spherical shell head model. Phys Med Biol 2002; 47(12):2179-85. 